\title{
A CONTINUATION METHOD FOR MOTION-PLANNING PROBLEMS*
}

\author{
YACINe Chitour ${ }^{1}$
}

\begin{abstract}
We apply the well-known homotopy continuation method to address the motion planning problem (MPP) for smooth driftless control-affine systems. The homotopy continuation method is a Newton-type procedure to effectively determine functions only defined implicitly. That approach requires first to characterize the singularities of a surjective map and next to prove global existence for the solution of an ordinary differential equation, the Wazewski equation. In the context of the MPP, the aforementioned singularities are the abnormal extremals associated to the dynamics of the control system and the Wazewski equation is an o.d.e. on the control space called the Path Lifting Equation (PLE). We first show elementary facts relative to the maximal solution of the PLE such as local existence and uniqueness. Then we prove two general results, a finite-dimensional reduction for the PLE on compact time intervals and a regularity preserving theorem. In a second part, if the Strong Bracket Generating Condition holds, we show, for several control spaces, the global existence of the solution of the PLE, extending a previous result of H.J. Sussmann.
\end{abstract}

Mathematics Subject Classification. 93B05, 93B29, 53C17, 34A12, 58C15.

Received October 1, 2004. Accepted May 26, 2005.

\section{INTRODUCTION}

In this paper, the motion planning problem for nonholonomic control systems is addressed. Consider the control system $\Sigma: \dot{x}=f(x, u)$, with $x \in M^{n}$, a smooth connected $n$-dimensional complete Riemannian manifold; $u=\left(u_{1}, \ldots, u_{m}\right)$ takes values in $\mathbb{R}^{m}$ and the class of admissible inputs $\mathcal{A}(\Sigma)$ is a space $\mathcal{H}$ of $\mathbb{R}^{m}$-valued functions defined on [0,1]. A trajectory of $\Sigma$ is an absolutely continuous curve $\gamma: J \rightarrow M^{n}$, with $J$ a subinterval of [0,1], such that there exists an admissible control $u \in \mathcal{A}(\Sigma)$ for which $\dot{\gamma}=f(\gamma, u)$, a.e. in $J$. Then $\Sigma$ is said to be completely controllable if, for every two points $p, q \in M$, there exists a trajectory $\gamma:[0,1] \rightarrow M^{n}$ of $\Sigma$ such that $\gamma(0)=p$ and $\gamma(1)=q$. In this case, the motion planning problem (MPP) is that of finding, for every pair $(p, q)$ of points of $M^{n}$, a control $u_{p, q}$ that steers $p$ to $q$.

Keywords and phrases. Homotopy continuation method, path following, Wazewski equation, sub-Riemannian geometry, nonholonomic control systems, motion planning problem.

* This work was part of the author's Ph.D. dissertation. The author would like to thank his former advisor Héctor J. Sussmann for many relevant ideas and advice.

1 Laboratoire des signaux et systèmes, Université de Paris-Sud, CNRS, Supélec, 91192, Gif-Sur-Yvette, France; yacine.chitour@lss.supelec.fr 
We might even want the trajectory of the control system to avoid obstacles: the motion planning problem with obstacle (MPPO) can be defined as follows: for $C$, a closed subset of $M^{n}$, the motion planning problem for $\Sigma$ on $M^{n}$ with obstacle $C$ consists of producing, for every $p, q$ in the same connected component of $M^{n} \backslash C$, a control $u_{p, q}$ that steers $p$ to $q$ so that the trajectory corresponding to $u_{p, q}$ is contained in $M^{n} \backslash C$.

The study of these subjects produced over the past few years many ingenious ideas and procedures, some of them making a systematic use of differential-geometric and differential-algebraic methods, e.g. nilpotent approximation [16], highly oscillatory inputs [20], optimal control [29], Pfaffian systems and Cartan prolongation [24] and differential flatness [22].

In [32], Sussmann proposed a differential-geometric approach for nonholonomic path-finding problems, based on the use of the homotopy continuation method (HCM). This method is widely used in several areas of mathematics, such as partial differential equations, bifurcation theory, differential geometry, differential topology (cf., for instance, Chow and Hale [9] and Hirsch [14]). Due to their robustness and simple implementation, numerical homotopy methods (or path following methods) turn out to be extremely useful in a large range of scientific applications ( $c f$. Allgower and Georg [2], Li [19] and Richter and Decarlo [27]). Conceived by Poincaré and systematically developed in the context of degree theory (Leray and Schauder [18]), the HCM consists in embedding a given problem $P$ into a parameterized family of problems $P_{s}$ and then, considering the solvability of $P_{s}$ as the parameter $s$ varies.

Let us briefly recall how the HCM works: let $M_{1}$ and $M_{2}$ be open subsets of $\mathbb{R}^{m_{1}}$ and $\mathbb{R}^{m_{2}}, m_{1}, m_{2} \geq 1, M_{2}$ connected and $\mathcal{E}: M_{1} \rightarrow M_{2}$ be a smooth mapping onto $M_{2}$. For $y \in M_{2}$ given, the objective is to determine $x \in M_{1}$ such that

$$
\mathcal{E}(x)=y
$$

Assuming that $\mathcal{E}$ is surjective, such an $x$ exists. Pick $x^{0} \in M_{1}$ and an smooth path $\pi:[0,1] \rightarrow M_{2}$ with $\pi(0)=\mathcal{E}\left(x_{0}\right)=y_{0}$ and $\pi(1)=y$. The next step consists in "lifting" $\pi$ to a smooth path $\Pi:[0,1] \rightarrow M_{1}$ such that

$$
\mathcal{E}(\Pi(s))=\pi(s), \quad s \in[0,1]
$$

If that procedure can be worked out, then $\Pi(1)$ is a solution of (1). Remark that in (2) $\Pi$ is only defined implicitly. One usually proceeds as follows: differentiate (2) to obtain

$$
D \mathcal{E}(\Pi(s)) \frac{\mathrm{d} \Pi}{\mathrm{d} s}(s)=\frac{\mathrm{d} \pi}{\mathrm{d} s}(s), \quad s \in[0,1], \Pi(0)=x_{0}
$$

If rank $D \mathcal{E}(\Pi(s))=m_{2}$ for $s \in[0,1]$, then $D \mathcal{E}(\Pi(s))$ has a right inverse $P(\Pi(s))$. Choosing $\frac{\mathrm{d} \Pi}{\mathrm{d} s}(s)$ as $P(\Pi(s))$. $\frac{\mathrm{d} \pi}{\mathrm{d} s}(s)$, leads to the following ordinary differential equation (o.d.e.)

$$
\frac{\mathrm{d} \Pi}{\mathrm{d} s}(s)=P(\Pi(s)) \cdot \frac{\mathrm{d} \pi}{\mathrm{d} s}(s) \quad s \in[0,1], \quad \Pi(0)=x_{0}
$$

Equation (3) is a Wazewski equation (cf. [25] or [33]). If it admits a global solution on [0,1], then (1) has a solution $y=\mathcal{E}(\Pi(1))$. To proceed rigorously, the issues to be overcome are:

(a) non degeneracy: if $S$ is the singular set of $\mathcal{E}$, it is necessary to have for $s \in[0,1]$, rank $D \mathcal{E}(\Pi(s))=m_{2}$ i.e. $\pi(s) \notin \mathcal{E}(S)$

(b) non-explosion: equation (3) must have a global solution $\Pi$ on $[0,1]$ with $\mathcal{E}(\Pi(0))=\pi(0)$.

The HCM does not always work if one of the two previous conditions fails to be satisfied. A simple example taken from Sussmann [32] shows the relevance of condition (a). Let $\mathcal{E}: \mathbb{R} \rightarrow \mathbb{R}$ be $\mathcal{E}(x)=x^{3}-3 x$. From 
$\mathcal{E}^{\prime}(x)=3\left(x^{2}-1\right)$, we get $S=\{-1,1\}$ and $\mathcal{E}(S)=\{-2,2\}$. Let $\pi:[0,1] \rightarrow \mathbb{R}$ be the path $\pi(t)=-3+6 t$. Then $\mathcal{E}(S) \subset \pi([0,1])=[-3,3]$ and the Wazewski equation is $\dot{z}=\frac{2}{z^{2}-1}$, which is defined for $z \in \mathbb{R} \backslash\{-1,1\}$. Clearly, its maximal solution is defined for $t \in[0,1 / 3)$ and $\Pi(t)$ approaches -1 as $t$ tends to $1 / 3$. Then $\pi$ cannot be globally lifted by using the HCM.

In the context of the MPP, the role of the surjective map $\mathcal{E}$ considered above is played by the end-point map $\mathcal{E}_{p, \mathcal{H}}: \mathcal{H} \rightarrow M$. To define it, we first assume that a No Explosion Condition (NEC) holds, i.e., for every $u \in \mathcal{H}$ and $p \in M$, the Cauchy problem $\dot{\gamma}=f(\gamma, u), \gamma(0)=p$, has a unique global solution $\gamma_{u, p}$ defined on $[0,1]$. We next fix an arbitrary point $p \in M$. Then, for every $u \in \mathcal{H}, \mathcal{E}_{p, \mathcal{H}}(u)$ is defined as $\gamma_{u, p}(1)$. The MPP can now be restated as the problem of finding a method producing for arbitrary points $p, q$ of $M^{n}$ a solution $u_{p, q}$ of the equation $\mathcal{E}_{p, \mathcal{H}}(u)=q$. Step $(a)$ requires the determination of the singularities of $\mathcal{E}_{p}$ (i.e. the controls $u$ for which rank $\left.D \mathcal{E}_{p, \mathcal{H}}(u)<n\right)$ and the choice of paths $\pi:[0,1] \rightarrow M^{n}$ which avoid the image of the singular set of $\mathcal{E}_{p, \mathcal{H}}$. Step (b) deals with (3), which is now an o.d.e. on $\mathcal{H}$ called the Path Lifting Equation (PLE), cf. [31].

We consider, in this work, driftless control-affine systems $\Sigma: \dot{x}=\sum_{i=1}^{m} u_{i} f_{i}(x)$ with $f=\left(f_{1}, \cdots, f_{m}\right)$ a finite sequence of smooth vector fields on $M^{n}$. Assuming that $\Sigma$ is completely controllable, the singularities of $\mathcal{E}_{p}$ are exactly the controls giving rise to the abnormal extremals of the distribution defined by $f$ (see [21] and references therein). The existence of nontrivial abnormal extremals leads to hard problems such as the determination of the precise structure of the singular set. As an example, the nature of the singular set of the Carnot group of rank two is still an open problem ( $c f$. Pansu [26]). For the MPP, it means that in general, Step $(a)$, is already difficult to achieve in general as far as concerns the non-degeneracy issue.

The connection between the HCM and the abnormal extremals was emphasized by Sussmann in [31], where it is shown that the HCM solves the MPP for control systems subject to the Strong Bracket Generating Condition (SBGC) with $\mathcal{H}=\mathcal{A}(\Sigma)=L^{2}\left([0,1], \mathbb{R}^{m}\right)$. This condition was tailored by Strichartz in [30] to precisely avoid the existence of nontrivial abnormal extremals. It is rather remarkable that when the SBGC holds, not only nontrivial abnormal do not exist but one can prove global existence of the solution of the PLE. In [6], another proof of Sussmann's result was provided and it was extended later to cover a case where nontrivial abnormal extremals occur [8]. There are other instances (of existence of nontrivial abnormal extremals) where one may lift enough paths $\pi$ in the state space using the PLE, in order to solve the MPP, [5,7]. Usually, the key step in showing global existence of the solution of the PLE consists of proving estimates on line-integrals along trajectories. For more application-oriented works in robotics, the HCM has been thoroughly used by Wen and his collaborators and we refer to the paper [10] and its bibliography for an account of that work.

In this paper, we present in full details the application of the HCM to driftless, control-affine systems (see also $[8,32]$ for a shorter presentation). In the next section, the PLE is precisely defined and local existence and uniqueness of its maximal solution are shown. Two general results for the PLE are then established. The first result puts forward a finite dimensional version of the PLE any compact interval $J$ in the domain of existence $I$ of the maximal solution $\Pi$ of the PLE. More precisely, consider a strictly increasing sequence $\left(\mathcal{H}^{j}\right)_{j \geq 1}$ of finite-dimensional subspaces of $\mathcal{H}$ such that $\bigcup_{j \geq 1} \mathcal{H}^{j}$ is dense in $\mathcal{H}$. Let $\Pi: I \rightarrow \mathcal{H}$ be the maximal solution of a PLE associated to some $p \in M$ and path $\pi$ avoiding the singular set $S$. Assume that $u_{0}=\Pi(0) \in \mathcal{H}_{0}$ and set $\mathcal{E}_{p, j}$, the end-point map associated to $p$ and $\mathcal{H}_{j}$. We prove that, for every compact subinterval $J=[0, a] \subset I$, the PLE defined by $\mathcal{E}_{p, j}$ and associated to $\pi$ has a global solution on $J$ for $j$ large enough. In particular, if $J=I=[0,1]$ (i.e. the PLE has a global solution), the previous result justifies the numerical implementations of the HCM, based in general on a Galerkin procedure. The second general result says that the maximal solution of the PLE is as regular as its initial condition, and so, independently of the control space $\mathcal{H}$ where the PLE is considered. For instance, if $\mathcal{H}=L^{2}\left([0,1], \mathbb{R}^{m}\right)$ and the initial condition $u_{0}$ is assumed to be continuous, then $\Pi$ will be continuous on its domain of existence. Section 3 is devoted to prove, in the case where the SBGC holds, global existence results for the PLE. In [31], that result was proved for $\mathcal{H}=L^{2}\left([0,1], \mathbb{R}^{m}\right)$. For completeness, we sketch an alternate proof (as given in [6]). We also consider the PLE on more regular input spaces. Those are subspaces of the standard Hilbert spaces $H^{k}\left([0,1], \mathbb{R}^{m}\right), k \geq 1$ so that the value of an input at time $t=1$ is zero. It means that trajectories of the resulting control system have zero speed at their ending point. The case of the MPP with obstacles is reduced to the classical MPP by a standard trick, the use of 
a potential function. Then every result obtained for the MPP of control affine-systems subject to the SBGC has an immediate corollary for the MPP with obstacles. Finally we gather some technical arguments in the Appendix.

\section{Presentation of the homotopy Continuation method}

\subsection{Notations and definitions}

Throughout this paper, the word "manifold" always means "finite-dimensional, Hausdorff, second countable manifold of class $C^{\infty}$ ". Let $M^{n}$ be a connected manifold of dimension $n \geq 1$, equipped with a complete Riemannian metric $\langle\cdot, \cdot\rangle$. Let us fix $x^{0} \in M^{n}$.

For $x \in M^{n}, T_{x} M^{n}$ and $T_{x}^{*} M^{n}$ denote the tangent and cotangent spaces of $M^{n}$ at $x$ respectively. We use $T M^{n}, T^{*} M^{n}, \pi$ and $\pi^{*}$ to denote the tangent and cotangent bundles of $M^{n}$ and the canonical projections from $T M^{n}$ and $T^{*} M^{n}$ onto $M^{n}$ respectively. Let $T^{\sharp} M \stackrel{\text { def }}{=}\left\{(x, z) \in T^{*} M^{n} ; z \in T_{x}^{*} M^{n}, z \neq 0\right\}, \pi^{\sharp}$, the corresponding projection onto $M^{n}$ and $\Omega$, the canonical symplectic form on $T^{*} M^{n}$.

We use the Riemannian metric $\langle\cdot, \cdot\rangle$ to identify $T_{x} M^{n}$ and its dual $T_{x}^{*} M^{n}$. For $x \in M^{n}, y \in T_{x} M^{n}$ and $z \in T_{x}^{*} M^{n},\|x\|$ denotes the geodesic distance between $x$ and $x^{0},\|y\|^{2}$ and $\|z\|^{2}$ denote $\langle y, y\rangle$ and $\langle z, z\rangle$ respectively. For each chart $\varpi=\left(x^{1}, \cdots, x^{n}\right): U \rightarrow \mathbb{R}^{n}$ on $M^{n}$ there are induced charts $T \varpi: \pi^{-1}(U) \rightarrow \mathbb{R}^{2 n}$, $T^{*} \varpi:\left(\pi^{*}\right)^{-1}(U) \rightarrow \mathbb{R}^{2 n}$ of $T M^{n}, T^{*} M^{n}$. A curve in $M^{n}$ is a continuous map $\gamma: I \rightarrow M^{n}$ defined on an interval $I$ of the real line. An arc in $M^{n}$ is an absolutely continuous curve whose domain is a compact interval. The boundary $\partial \gamma$ of an arc $\gamma:[a, b] \rightarrow M^{n}$ is the ordered pair $\partial \gamma=(\gamma(a), \gamma(b))$. If $X$ is a subset of $M$, we use $\bar{X}$ and $\stackrel{\circ}{X}$ to denote respectively the closure and the interior of $X$.

We use $V^{k}\left(M^{n}\right)\left(V^{k}\left(T^{*} M^{n}\right)\right.$ resp.) to denote the set of vector fields of class $C^{k}$ on $M^{n}$ ( $T^{*} M^{n}$ resp.). We use $D X$ to denote the Jacobian of $X$, i.e., the differential of $X$. (In coordinates, it can be identified with a square matrix whose columns are the partial derivatives $\left.\frac{\partial X}{\partial x^{1}}, \cdots, \frac{\partial X}{\partial x^{n}} \cdot\right)$ For $X \in V^{l}\left(M^{n}\right)(l \geq 1)$, the variational covector field $T^{*} X$ of $X$ is the element of $V^{l-1}\left(T^{*} M\right)$ expressed in local coordinates of $T^{*} M$ by

$$
\left(T^{*} X\right)(x, z)=(X(x),-z D X(x)) .
$$

If $f$ is an $m$-tuple of elements of $V^{l}\left(M^{n}\right)(l \geq 1)$, i.e. $f=\left(f_{1}, \cdots, f_{m}\right)$, we use $T^{*} f$ to denote the $m$-tuple of elements of $V^{l-1}\left(T^{*} M\right)$ given by $\left(T^{*} f_{1}, \cdots, T^{*} f_{m}\right)$. Clearly, $T^{*} X$ is intrinsically defined (i.e. independent on the choice of a chart $\varpi)$.

The Hamiltonian function corresponding to a vector field $X$ on $M$ is the function $H_{X}: T^{*} M^{n} \rightarrow \mathbb{R}$ given by $H_{X}(x, z)=\langle z, X(x)\rangle$, for $x \in M^{n}$ and $z \in T_{x}^{*} M$. Then $H_{X}$ is of class $C^{k}$ if and only if $X$ is. The Hamiltonian Vector Field associated to a function $H: T^{*} M^{n} \rightarrow \mathbb{R}$ of class $C^{k}, k \geq 1$ is the vector field $\vec{H} \in V^{k-1}\left(T^{*} M^{n}\right)$ defined by $\Omega(\vec{H}(x, z), w)=\mathrm{d} H(w)$ for all $w \in T_{(x, z)} T^{*} M^{n}$. In coordinates, $\vec{H}$ is given by $\vec{H}(x, z)=\left(\left(\frac{\partial H}{\partial z}\right)^{T},-\frac{\partial H}{\partial x}\right)$, where " $T$ " denotes transpose. If $X \in V^{1}\left(M^{n}\right), T^{*} X$ is just $\vec{H}_{X}$ (which is therefore intrinsically defined).

For $a \leq b \in[0,1]$ and $k$ integer, let $H^{k}\left([a, b], \mathbb{R}^{m}\right)$ of $m$-tuples $u_{i} \in H^{k}(a, b), 1 \leq i \leq m$. Recall that a real valued function $w$ belongs to $H^{k}(a, b)$ if $\left.w \in L^{2}(a, b]\right)$ together with its derivatives of order $\leq k$, with the convention that $H^{0}=L^{2}$. Finally, for $k \geq 0$, let $C^{k}\left([0,1], \mathbb{R}^{m}\right)$ be the function space of $m$-tuples $u=$ $\left(u_{1}, \cdots, u_{m}\right)$, where $u_{i}, 1 \leq i \leq m$, is $k$-times continuously differentiable. The spaces $H^{k}\left([a, b], \mathbb{R}^{m}\right)$ are Hilbert spaces for their standard inner products $(u, v)_{H^{k}(a, b)}=\sum_{j=0}^{k}\left(u^{(j)}, v^{(j)}\right)_{L^{2}(a, b)}$, where $(u, v)_{L^{2}(a, b)} \stackrel{\text { def }}{=}$ $\sum_{i=1}^{m} \int_{a}^{b} u_{i}(t) v_{i}(t) \mathrm{d} t$. When $[a, b]=[0,1],[a, b]$ is simply dropped. If $\mathcal{H}$ is a closed linear subspace of $H^{k}(0,1)$, $k \geq 0$, we use $\mathcal{H}_{m}$ to denote the closed linear subspace of $H^{k}\left([0,1], \mathbb{R}^{m}\right)$ of $m$-tuples of elements of $\mathcal{H}$. For $u, v \in \mathcal{H}_{m}$, we use $(u, v)_{\mathcal{H}_{m}}$ to denote the Hilbert product between $u$ and $v$.

For $r \geq 1$, let $\mathcal{H}^{r, 1}$ be the subspace of functions $u \in \mathcal{H}^{r}([0,1])$ subject to

$$
u(1)=\cdots=u^{(r-1)}(1)=0, \quad\|u\|_{\mathcal{H}^{r, 1}} \stackrel{\text { def }}{=}\left\|u^{(r)}\right\|_{L^{2}} .
$$


The controls are Lebesgue integrable functions $u:[a, b] \rightarrow \mathbb{R}^{m}$ with $[a, b] \subset[0,1]$ (depending in general on $u$ ). We denote that class of controls by $\mathcal{A}_{m}$.

In the sequel, we will consider bounded linear operators $L: \mathcal{H} \rightarrow \mathcal{F}$ where $\left(\mathcal{H},(\cdot, \cdot)_{\mathcal{H}}\right)$ and $\left(\mathcal{F},(\cdot, \cdot)_{\mathcal{F}}\right)$ are Hilbert spaces. We use $L^{T}: \mathcal{F} \rightarrow \mathcal{H}$ to denote the adjoint operator associated to $L$, i.e., the unique linear operator verifying,

$$
(L v, w)_{\mathcal{F}}=\left(v, L^{T} w\right)_{H},
$$

for every $v \in \mathcal{H}$ and $w \in \mathcal{F}$. Sometimes, in order to emphasize the dependence of $L^{T}$ with respect to $\mathcal{H}$, we we will use instead $L^{T_{\mathcal{H}}}$ to denote the adjoint operator associated to $L$.

A driftless control-affine system $\Sigma$ is defined by the 4 -tuple $\left(M^{n}, \mathbb{R}^{m}, \mathcal{A}(\Sigma), f\right)$, where $\dot{x}=\sum_{i=1}^{m} u_{i} f_{i}(x)$, $x \in M^{n}, u=\left(u_{1}, \ldots, u_{m}\right) \in \mathbb{R}^{m}, f=\left(f_{1}, \cdots, f_{m}\right)$ is an $m$-tuple of elements of $V^{\infty}\left(M^{n}\right)$ and a $\Sigma$-admissible control $u$ (or simply admissible when there is no confusion on the control system) is in $\mathcal{A}(\Sigma), \mathcal{A}(\Sigma) \subset \mathcal{A}_{m}$. (For more general definition of a control system $c f$. Jurdjevic [15].)

A trajectory of $\Sigma$ generated by the admissible control $u$ defined on $[a, b]$ is an $\operatorname{arc} \gamma: J \rightarrow M^{n}$ such that $J$ is a subinterval of $[a, b]$ and

$$
\dot{\gamma}(t)=\sum_{i=1}^{m} u_{i}(t) f_{i}(\gamma(t)), \quad \text { a.e. in } J .
$$

Notice that the time-varying vector field $(x, t) \rightarrow \sum_{i=1}^{m} u_{i}(t) f_{i}(x(t))$ arising from $u$ satisfies the conditions of the Carathéodory existence theorem (cf. [21]). Then, given $x_{0} \in M^{n}$, there exists a unique maximal trajectory of $\Sigma, \gamma_{u, x_{0}}$, generated by $u$ such that, $\gamma_{u, x_{0}}(a)=x_{0}$ and $\gamma_{u, x_{0}}$ is defined on a subinterval $J \subset[a, b]$ containing $a$ and relatively open in $[a, b]$.

To a control system $\Sigma$ is associated the collection $T R(\Sigma)$ of all the trajectories generated by the admissible controls. For any family of controls $O \subset \mathcal{A}(\Sigma)$, the system $\Sigma$ is $O$-complete ( $c f$. Sontag [28]) if, for every element $u:[a, b] \rightarrow \mathbb{R}^{m}$ of $O$ and every $x_{0} \in M^{n}$, the maximal trajectory $\gamma_{u, x_{0}}$ generated by $u$ is defined on $[a, b]$. If $O=\mathcal{A}(\Sigma)$, we say that the system is complete or that the No Explosion Condition (NEC) holds for $\Sigma$.

A control system $\Sigma$ is completely controllable if for every two points $p, q \in M^{n}$ there exists an element $\gamma:[a, b] \rightarrow M^{n}$ of $T R(\Sigma)$ so that $\gamma(a)=p$ and $\gamma(b)=q$. Let $L_{\Sigma}$ be the Lie algebra of vector fields on $M^{n}$ generated by $f_{1}, \ldots, f_{m}$. The Lie-Algebra rank condition (LARC) holds for $\Sigma$ if

$$
\forall x \in M^{n}, L_{\Sigma}(x) \stackrel{\text { def }}{=}\left\{X(x): X \in L_{\Sigma}\right\} \text { is equal to } T_{x} M^{n} .
$$

Recall that if the system $\Sigma$ satisfies the LARC, then $\Sigma$ is completely controllable (cf. Jurdjevic [15]). The control system $\Sigma$ satisfies the Strong Bracket Generating Condition (SBGC) (cf. Strichartz [30]) if

$$
\begin{aligned}
& \forall \theta \in \mathbb{R}^{m} \backslash\{0\}, \forall x \in M, \theta \cdot f \stackrel{\text { def }}{=} \sum_{i=1}^{m} \theta_{i} f_{i} \text { if } \\
& \text { the vectors } f_{1}(x), \cdots, f_{m}(x),\left[\theta \cdot f, f_{1}\right](x), \cdots,\left[\theta \cdot f, f_{m}\right](x) \operatorname{span} T_{x} M .
\end{aligned}
$$

If the LARC or SBGC hold for $f_{i}, i=1, \ldots, m$, it still holds for $\bar{f}_{i}=\psi f_{i}, i=1, \ldots, m$, where $\psi$ is an arbitrary nowhere vanishing $C^{\infty}$ real-valued function.

Given a control system $\Sigma=\left(M^{n}, \mathbb{R}^{m}, \mathcal{A}_{m}, f\right)$ and $\mathcal{H}_{m} \subset \mathcal{A}_{m}$ for some closed linear subspace $\mathcal{H}$ of $H^{k}([0,1])$, $k \geq 0$, we want to transform $\Sigma$ into another control system $\bar{\Sigma}$ so that

(C1) $\mathcal{A}(\bar{\Sigma})=\mathcal{H}_{m}$ and $\bar{\Sigma}$ is complete;

$(C 2) \bar{\Sigma}$ is completely controllable;

(C3) $T R(\bar{\Sigma}) \subset T R(\Sigma)$.

It is clear that if $f$ satisfies a growth condition of the type

$$
\exists A, B>0, \forall x \in M^{n} \quad\left\|f_{i}(x)\right\| \leq A\|x\|+B,
$$

for $i=1, \ldots, m$, then $\Sigma$ is $\mathcal{H}_{m}$-complete. In addition the control space $\mathcal{H}$ must verify some kind of property to insure condition $(C 2)$. For that purpose, Property $(C l)_{k}$ (introduced first in [12]) is given in Definition 3, 
Section 5.1. It turns out that the usual $H^{k}$ and the $\mathcal{H}^{r, 1}$ satisfy it (see Sect. 5.1 for a proof). Note that (6) is independent of a particular choice of $\mathcal{H}$ satisfying property $(C l)_{k}, k \geq 0$. Let $\bar{\Sigma} \stackrel{\text { def }}{=}\left(M^{n}, \mathbb{R}^{m}, \mathcal{H}_{m}, \bar{f}\right)$ where $\bar{f}_{i}=\psi f_{i}, i=1, \ldots, m, \psi=\frac{1}{1+\sum_{i=1}^{m}\left\|f_{i}\right\|^{2}}$. The vector fields $\left(\bar{f}_{1}, \cdots, \overline{f_{m}}\right)$ are then bounded and, since $M^{n}$ is complete, condition $(C 1)$ follows. In addition, if the (LARC) holds for $\Sigma$, it also holds for $\bar{\Sigma}$ and condition $(C 2)$ is valid ( $c f$. Cor. 4.7 of [12]). As for condition (C3), this is a simple consequence of the definition of $\bar{\Sigma}$.

Definition 1. A control system $\Sigma=\left(M^{n}, \mathbb{R}^{m}, \mathcal{H}_{m}, f\right)$ is said to be CC-tempered if the LARC and (6) hold and if $\mathcal{H}_{m}$ is a Hilbert space satisfying Property $(\mathrm{Cl})_{k}, k \geq 0$. In that case, $\Sigma$ is complete and completely controllable.

Notice that the LARC and (6) are properties of the sole $m$-tuple of vector fields, $f$. If they hold for a control system $\Sigma=\left(M^{n}, \mathbb{R}^{m}, \overline{\mathcal{H}}_{m}, f\right)$ then, for every $\mathcal{H}$ satisfying Property $(C l)_{k}$ for some $k \geq 0$, the control system $\Sigma_{\mathcal{H}}=\left(M^{n}, \mathbb{R}^{m}, \mathcal{H}, f\right)$ is also CC-tempered. In particular, $\Sigma_{\mathcal{H}}$ is complete and completely controllable. For simplicity, we will write $M$ for $M^{n}$ and $\mathcal{H}$ for $\mathcal{H}_{m}$ when the context is clear.

Let $\Sigma=\left(M, \mathbb{R}^{m}, \mathcal{A}(\Sigma), f\right)$. The Hamiltonian lift of $\Sigma$ is $T^{*} \Sigma \stackrel{\text { def }}{=}\left(T^{*} M, \mathbb{R}^{m}, \mathcal{A}(\Sigma), T^{*} f\right)$ with

$$
\dot{\xi}=\sum_{i=1}^{m} u_{i} T^{*} f_{i}(\xi)
$$

For $u \in \mathcal{H}$, a trajectory $\xi$ of $T^{*} \Sigma$ is a pair $(\gamma, \lambda)$, with $\gamma$ a trajectory of $\Sigma$ generated by $u$ and $\lambda$ the field of covectors along $\gamma\left(\right.$ i.e. $\lambda(t) \in T_{\gamma(t)}^{*} M$ for $\left.t \in[0,1]\right)$ such that $\lambda$ is an adjoint vector along $(\gamma, \lambda)$, i.e. it satisfies the adjoint equation along $\gamma$,

$$
\dot{\lambda}=-\lambda\left(\sum_{i=1}^{m} u_{i} D f_{i}(\gamma(t))\right), \quad \text { a.e. in }[0,1]
$$

The adjoint vector $\lambda$ is nontrivial if it does not vanish on $[0,1]$.

For any $r$-tuple $g=\left(g_{1}, \ldots, g_{r}\right)$ of elements of $V^{l}(M), r, l \geq 0$, and $\psi: M \rightarrow \mathbb{R}$ of class $C^{1}$, the $g$-Lie derivative of $\psi$ is given by $\nabla_{g} \psi=\left(g_{1} \cdot \psi, \cdots, g_{r} \cdot \psi\right)^{T}: M \rightarrow \mathbb{R}^{r}$. The time derivative of $\psi$ along a trajectory $\gamma$ of $\Sigma$ corresponding to a control $u=\left(u_{1}, \ldots, u_{m}\right)$ is

$$
\frac{\mathrm{d}}{\mathrm{d} t} \psi(\gamma(t))=\mathrm{d} \psi(\gamma(t))(\dot{\gamma}(t))=\sum_{i=1}^{m} u_{i}(t)\left(f_{i} \cdot \psi\right)(\gamma(t)) \stackrel{\text { def }}{=}\left(u \cdot \nabla_{f} \psi\right)(\gamma(t))
$$

where $f=\left(f_{1}, \cdots, f_{m}\right)$. Similarly, for $f=\left(f_{1}, \cdots, f_{m}\right)$, an $m$-tuple of elements of $V^{l}(M)$, and $u=\left(u_{1}, \cdots, u_{m}\right)$ in $\mathcal{H}$, we write $\sum_{i=1}^{m} u_{i}(t) f_{i}(\gamma(t)), \sum_{i=1}^{m} u_{i}(t) D f_{i}(\gamma(t))$ and $\sum_{i=1}^{m} u_{i}(t) T^{*} f_{i}(\gamma(t))$ resp. as $(u \cdot f)(\gamma(t)),(u$. $D f)(\gamma(t))$ and $\left(u \cdot T^{*} f\right)(\gamma(t))$ resp. Using these notations, we write (5), (8) and (7) as $\dot{\gamma}(t)=(u \cdot f)(\gamma(t))$, $\dot{\lambda}(t)=-\lambda(t)(u \cdot D f)(\gamma(t))$ and $\dot{\xi}(t)=\left(u \cdot T^{*} f\right)(\xi(t))$.

\subsection{Study of the end-point map $\mathcal{E}$}

Let $\Sigma$ be a CC-tempered control system and $x_{0}$ be a point of $M$. Recall that the end-point map (EM) $\mathcal{E}_{x^{0}, \mathcal{H}}$ (or simply $\mathcal{E}$ ) : $\mathcal{H} \rightarrow M$ is defined by $\mathcal{E}(u)=\gamma_{u}(1)$, where $\gamma_{u}$ is the solution of $(5)$ with $\gamma_{u}(0)=x^{0}$. Since $\Sigma$ is complete and completely controllable, $\mathcal{E}$ is surjective. In addition, $\mathcal{E}$ is Fréchet-differentiable and, for $u \in \mathcal{H}$, we have

$$
\begin{aligned}
D \mathcal{E}(u): \quad & \mathcal{H} \rightarrow T_{\mathcal{E}(u)} M \\
& v \mapsto D \mathcal{E}(u) \cdot v=y_{v}(1),
\end{aligned}
$$


where, for every $v \in \mathcal{H}, y_{v}:[0,1] \rightarrow T M$ is the solution of the variational equation

$$
\dot{y}(t)=(u \cdot D f)\left(\gamma_{u}(t)\right) y(t)+(v \cdot f)\left(\gamma_{u}(t)\right) \text { a.e. in }[0,1], \quad y(0)=0 .
$$

Let $\mathcal{E}_{t}^{u}$ be the flow associated to $(5)$, i.e., for $t \in[0,1]$ and $x \in M, \mathcal{E}_{t}^{u}(x) \stackrel{\text { def }}{=} \gamma_{u, x}(t)$, where $\gamma_{u, x}(t)$ is the solution of (5) starting at $x$. We use $\left(\mathcal{E}_{t}^{u}\right)_{*}(x)$ to denote the Fréchet differential of $\mathcal{E}_{t}^{u}$ at the point $x \in M$, so that $\left(\mathcal{E}_{t}^{u}\right)_{*}(x)$ is a linear map from $T_{x} M$ to $T_{\gamma_{u, x}(t)} M$ with transpose map $\left(\mathcal{E}_{t}^{u}\right)^{*}$. Then,

$$
D \mathcal{E}(u) \cdot v=y_{v}(1)=\int_{0}^{1} \sum_{i=1}^{m} v_{i}(t)\left(\mathcal{E}_{1}^{u}\right)_{*}\left(\mathcal{E}_{t}^{u}\right)_{*}^{-1}\left(f_{i}\left(\gamma_{u, x}(t)\right)\right) \mathrm{d} t .
$$

For $z$ in $T_{\mathcal{E}(u)}^{*} M$ and $v \in \mathcal{H}$, then $\langle z, D \mathcal{E}(u) \cdot v\rangle=\left(D \mathcal{E}(u)^{T_{\mathcal{H}}} z, v\right)_{\mathcal{H}}$ and for $y \in T_{\gamma_{u, x}(t)} M,\left\langle z,\left(\left(\mathcal{E}_{1}^{u}\right)_{*}\left(\mathcal{E}_{t}^{u}\right)_{*}^{-1} y\right\rangle=\right.$ $\left\langle\left(\left(\mathcal{E}_{t}^{u}\right)^{*}\right)^{-1}\left(\mathcal{E}_{1}^{u}\right)^{*} z, y\right\rangle$. Then $\left(\left(\mathcal{E}_{t}^{u}\right)^{*}\right)^{-1}\left(\mathcal{E}_{1}^{u}\right)^{*} z$ is the adjoint vector $\lambda(t)$ along $\gamma_{u}$, with $\lambda(1)=z$.

For $i=1, \cdots, m$, we use $\varphi_{i, z}(t), t \in[0,1]$, to denote the Switching Functions (SF) of $\Sigma$, i.e. the value of the Hamiltonian function $H_{f_{i}}$ along the trajectory $\left(\gamma_{u}, \lambda\right)$ of $T^{*} \Sigma$. We have

$$
\varphi_{i, z}(t)=\left\langle\lambda(t), f_{i}\left(\gamma_{u}(t)\right)\right\rangle, i=1, \ldots, m, t \in[0,1], \varphi_{i, z}(1)=\left\langle z, f_{i}(\mathcal{E}(u))\right\rangle .
$$

The switching vector is defined as next,

$$
\varphi_{z}(t) \stackrel{\text { def }}{=}\left(\varphi_{i, z}(t)\right)_{i=1, \cdots, m}
$$

and, for any subinterval $[a, b]$ of $[0,1],\left\|\varphi_{z}\right\|_{L^{2}([a, b])}^{2} \stackrel{\text { def }}{=} \int_{a}^{b} \sum_{i=1}^{m} \varphi_{i, z}^{2}(t) \mathrm{d} t$.

Using (11), we get

$$
\langle z, D \mathcal{E}(u) \cdot v\rangle=\int_{0}^{1} \sum_{i=1}^{m} v_{i}(t) \varphi_{i, z}(t) \mathrm{d} t=\left(\varphi_{z}, v\right)_{L^{2}}
$$

Therefore, $D \mathcal{E}(u)^{T_{\mathcal{H}}} z$ is the $\mathcal{H}$-representation of $\varphi_{z}$, i.e. for $v \in \mathcal{H},\left(D \mathcal{E}(u)^{T_{\mathcal{H}}} z, v\right)_{\mathcal{H}}=\left(\varphi_{z}, v\right)_{L^{2}}$.

In particular, if $\mathcal{H}=L^{2}\left([0,1], \mathbb{R}^{m}\right)$, we have

$$
D \mathcal{E}(u)^{T_{\mathcal{H}}} z=\varphi_{z}
$$

For $u \in \mathcal{H}$, consider the non-negative symmetric matrix

$$
G(u)=D \mathcal{E}(u) D \mathcal{E}(u)^{T_{\mathcal{H}}},
$$

the controllability Gramian of (9). Then the following fundamental relations hold

$$
\forall z \in T_{\mathcal{E}(u)}^{*} M,\langle z, G(u) z\rangle=\left\|D \mathcal{E}(u)^{T_{\mathcal{H}}} z\right\|_{\mathcal{H}}^{2}, \operatorname{rank} D \mathcal{E}(u)=n<=>G(u)>0 .
$$

The singular set $S$ is the set of singular controls i.e. $S \stackrel{\text { def }}{=}\{u \in \mathcal{H}$; rank $D \mathcal{E}(u)<n\}$ and $\mathcal{E}(S)$ is the singular value set. Clearly $u \in S$ if and only if there exists some nonzero $z$ in $T_{\mathcal{E}(u)}^{*} M$ such that

$$
\forall i=1, \ldots, m, \quad\left\langle\lambda_{z}(t), f_{i}\left(\gamma_{u}(t)\right)\right\rangle=\varphi_{i, z}(t) \equiv 0 \text { in }[0,1]
$$

i.e., if and only if there exists a nontrivial adjoint vector $\lambda_{z}$ along $\gamma_{u}$ such that the corresponding SF are identically equal to zero on $[0,1]$. In this case, $\gamma_{u}(t)$ is said to be an abnormal trajectory, according to the optimal control terminology ( $c f$. Lee and Markus [17] for example). If the $f_{i}$ 's are linearly independent everywhere on $M$ and, given an initial condition for (5), we have a one-to-one correspondence between the controls $u$ and the corresponding trajectories $\gamma_{u}$. For this reason, we call a control corresponding to an abnormal trajectory $\gamma_{u}$ an abnormal control (AC). 
Remark 1. Since $S=\{u \in \mathcal{H}$, $\operatorname{det} G(u)=0\}$, it is a closed subset of $\mathcal{H}$. Furthermore, when $m<n$, the zero control is abnormal. For general nonholonomic problems, nontrivial abnormal controls can occur (cf. e.g. Liu and Sussmann [21], Montgomery [23]). However, when the SBGC holds, it turns out that $S$ reduces to $\{0\}$ ( $c f$. Strichartz [30]).

Let $u \in \mathcal{H}$ and $\xi=(\gamma, \lambda)$ a corresponding trajectory of $T^{*} \Sigma$ such that $\lambda(1)=z \in T_{\mathcal{E}(u)}^{*} M$. For any vector field $B \in V^{1}(M)$ and $t \in[0,1]$, set $\varphi_{B, z}(t):=\varphi_{B, z}(t)=\left\langle\lambda(t), B\left(\gamma_{u}(t)\right)\right\rangle$. If $B$ is not equal to any $f_{i}, 1 \leq i \leq m$, as elements of $V^{1}(M)$, then $\varphi_{B, z}$ is called a Pseudo-Switching Function (PSF). The time derivative of $\varphi_{B, z}$ is given by

$$
\dot{\varphi}_{B, z}(t)=\sum_{j=1}^{m} u_{j}(t)\left\langle\lambda(t),\left[f_{j}, B\right]\left(\gamma_{u}(t)\right)\right\rangle=\sum_{j=1}^{m} u_{j}(t) \varphi_{\left[f_{j}, B\right], z}(t), \quad \text { a.e. in }[0,1] .
$$

As a consequence, we obtain the following formula for the derivatives of the SF: for $i=1, \ldots, m$,

$$
\dot{\varphi}_{z}(t)=\mathcal{A} u(t), \quad \text { a.e. in }[0,1]
$$

where $\mathcal{A}$ is the skew-symmetric matrix $\mathcal{A}=\left(\varphi_{\left[f_{j}, f_{i}\right], z}\left(\gamma_{u}(t)\right)_{i, j=1 \cdots, m}\right.$. If the LARC holds, then for any compact $K$ of $M$, there exist an integer $r \geq m$ and a finite subset $\left\{f_{1}, \cdots, f_{m}, f_{m+1}, \cdots f_{r}\right\}$ of $L_{\Sigma}$, with $f_{m+1}, \ldots, f_{r}$ iterated Lie brackets of the $f_{i}$ 's, such that

$$
\forall x \in K, \operatorname{span}\left(f_{1}(x), \ldots, f_{m}(x), f_{m+1}(x), \ldots, f_{r}(x)\right)=T_{x} M
$$

Then, (see Sect. 5.2), there exists an open bounded subset $U_{K}$ of $M$ containing $K$ such that if $B \in V^{\infty}(M)$ there exist $r C^{\infty}$ functions $v_{1, B}, \cdots, v_{r, B}$ defined on $U_{K}$ with

$$
\forall x \in U_{K}, B(x)=\sum_{i=1}^{r} v_{i, B}(x) f_{i}(x) .
$$

Apply $(20)$ with $K=\gamma_{u}([0,1])$ and $B \in L_{\Sigma}$. Then, $B\left(\gamma_{u}(t)\right)=\sum_{i=1}^{r} v_{i, B}\left(\gamma_{u}(t)\right) f_{i}\left(\gamma_{u}(t)\right)$. This implies that $\left(v_{i, B}(t) \stackrel{\text { def }}{=} v_{i, B}\left(\gamma_{u}(t)\right)\right)$

$$
\varphi_{B, z}(t)=\left\langle\lambda_{z}(t), B\left(\gamma_{u}(t)\right)\right\rangle=\sum_{i=1}^{r} v_{i, B}(t)\left\langle\lambda_{z}(t), f_{i}\left(\gamma_{u}(t)\right)\right\rangle=\sum_{i=1}^{r} v_{i, B}(t) \varphi_{f_{i}, z}(t) .
$$

Using (21), we write (17) for $B=f_{i}, 1 \leq i \leq r$, as

$$
\begin{aligned}
\dot{\varphi}_{f_{i}, z}(t) & =\sum_{j=1}^{m} u_{j}(t) \varphi_{\left[f_{j}, f_{i}\right], z}(t)=\sum_{j=1}^{m} u_{j}(t)\left(\sum_{q=1}^{r} v_{q,\left[f_{j}, f_{q}\right]}(t) \varphi_{f_{q}, z}(t)\right) \\
& =\sum_{q=1}^{r}\left(\sum_{j=1}^{m} u_{j}(t) v_{q,\left[f_{j}, f_{q}\right]}(t)\right) \varphi_{f_{q}, z}(t)=\sum_{q=1}^{r} w_{q, i}(t) \varphi_{f_{q}, z}(t), \text { a.e. in }[0,1],
\end{aligned}
$$

where $w_{q, l}(t)=\sum_{j=1}^{m} u_{j}(t) v_{q,\left[f_{j}, f_{l}\right]}(t)$. The following proposition holds:

Proposition 1. For $u \in \mathcal{H}$ there exists $r \geq 0$ such that, for $z \in T_{\mathcal{E}(u)}^{*} M$, the corresponding SF together with suitable PSF, satisfy a first order linear differential system of dimension $r$.

If the SBGC holds, then the integer $r$ defined in Proposition 1 can be taken independent of $u$. Actually, one can choose $r$ to be $2 m$. 
We finally treat the MPP with obstacles (MPPO for short) by the use of a potential function $\psi$ defined next. An obstacle $C$ is a nonempty closed subset $C$ of $M$ such that $\widetilde{M}=M \backslash C$ is also nonempty. Then, there exists a smooth bounded function $\psi: M \rightarrow \mathbb{R},\left(c f\right.$. Sect. 5.3) such that $\psi>0$ on $\tilde{M}, \psi=0$ on $C$ and and $\left(\widetilde{M},\langle\langle\cdot, \cdot\rangle\rangle_{x}\right)$ is a complete Riemaniann manifold, where

$$
\langle\langle\cdot, \cdot\rangle\rangle_{x}=\frac{\langle\cdot, \cdot\rangle_{x}}{\psi^{2}}
$$

Let $\Sigma=\left(M, \mathbb{R}^{m}, \mathcal{H}, f\right)$ be a CC-tempered control system and $\Sigma_{0}$, the control system on $M$ defined by

$$
\dot{y}=\sum_{i=1}^{m} v_{i} \bar{f}_{i}(y), m \geq 1,
$$

where $\bar{f}_{i}=\psi f_{i}, i=1, \cdots, m$. Then the vector fields $\bar{f}_{i}, i=1, \cdots, m$ satisfy the NEC. Furthermore, $\widetilde{M}$ is invariant under the control system $\Sigma_{0}$. More precisely, we have

Lemma 1. $\forall v \in \mathcal{H}, \forall p \in \widetilde{M}$, if $\tilde{\gamma}_{v}$ is the solution of $(24)$ with $\tilde{\gamma}(0)=p$, then $\tilde{\gamma}([0,1]) \subset \widetilde{M}$.

Proof of Lemma 1. If not, then there exist $x_{0} \in \widetilde{M}, v_{0} \in \mathcal{H}$ and $t_{1} \in[0,1]$ with $\tilde{\gamma}\left(t_{1}\right)=q \in \partial C$, where $\tilde{\gamma}_{v_{0}}$ is the solution of $(24)$ with $\tilde{\gamma}(0)=x_{0}$. On the other hand, for every $w \in \mathcal{H}$ the solution of (24) corresponding to $w$ with initial condition $q$ is the constant path $q$. Hence, $\tilde{\gamma}_{v_{0}} \equiv q$, which is a contradiction since $x_{0} \neq q$.

For every $t \in[0,1], \tilde{\gamma}(t)$ belongs to the connected component of $\widetilde{M}$ containing $p$. Let $\bar{f}=\left(\overline{f_{1}}, \cdots, \overline{f_{m}}\right)$. For every connected component $C$ of $\tilde{M}$, Lemma 1 implies that $\Sigma_{C}=\left(C, \mathbb{R}^{m}, \mathcal{H}, \bar{f}\right)$ is a CC-tempered control system. Thus, the MPPO is reduced to a MPP for each connected component of $\tilde{M}$. On one hand, the LARC and the SBGC are preserved but, on the other hand, the Lie bracket relations of the vector fields associated to the original system will in general be different after this reduction.

\subsection{Application of the HCM to the MPP}

For the rest of the paper, all considered control systems are supposed to be CC-tempered, unless otherwise indicated.

We start with the following remark.

Remark 2. Suppose there exists an open neighborhood $M_{0}$ of some $x_{0}$ in $M$ such that, for every $x \in M_{0}$, the rank of the subspace of $T_{x} M$ spanned by $f_{1}(x), \cdots, f_{m}(x)$ is equal to $n$. With no loss of generality, we can assume that $M_{0}$ is an open ball of $\mathbb{R}^{n}$ centered at $x_{0}=0$. Then, the MPP is trivially solved at least locally: every path $\pi$ contained in $M_{0}$ can be regarded as a trajectory of $\Sigma$. Indeed, let $\pi:[0,1]$ be a $C^{1}$ path contained in $M_{0}$. If we use $F(x)$, the $m \times n$ matrix with $f_{i}$ as column vectors, and $G(x)$ a smooth right inverse of $F(x)$, then, for $t \in[0,1] \dot{\pi}=F(\pi(t)) G(\pi(t)) \dot{\pi}=\sum_{i=1}^{m} v_{i}(t) f_{i}(\pi(t))$, for $m$ continuous functions $v_{i}:[0,1] \rightarrow \mathbb{R}$, $i=1, \cdots, m$.

From now on, $m<n$ and, for every $x \in M$, the $f_{i}(x)$ are linearly independent in $T_{x} M$.

Pick $x_{0} \in M$ and let $\mathcal{E}: \mathcal{H} \rightarrow M$ be the corresponding end-point map. The application of the HCM to the MPP is divided into two steps.

Step 1. Determine the singular set $S$ and $\mathcal{E}(S)$, the singular value set.

The determination of $S$ can proceed as follows: let $u$ be a nonzero abnormal control giving rise, for every $z \in T_{\mathcal{E}(u)}^{*} M$, to a trajectory $\xi=\left(\gamma_{u}, \lambda\right)$ of $T^{*} \Sigma$. According to Proposition 1 , the corresponding SF, $\varphi_{i, z}, i=$ $1, \cdots, m$, together with suitable PSF, $\varphi_{i, z}, i=m+1, \cdots, r$ satisfy a first order linear differential system $(D S)_{z}$ of dimension $r$. There exists $z \in T_{\mathcal{E}(u)}^{*} M,\|z\|=1$, such that $\varphi_{i, z} \equiv 0, i=1, \cdots, m$. Applying (18) for each $\mathrm{SF}$, we obtain $m$ equations involving the $\operatorname{PSF} \varphi_{\left[f_{i}, f_{j}\right], z}, i, j=1, \cdots, m$. Using (19), we get $m$ equations only 
involving the PSF $\varphi_{i, z}, i=m+1, \cdots, r$. In addition, $(D S)_{z}$ is reduced to another first order linear differential system $(D S)_{z}^{\prime}$ of dimension $r-m$ satisfied by the PSF $\varphi_{i, z}, i=m+1, \cdots, r$ only. Then, a control $u$ is abnormal if there exists $z \in T_{\mathcal{E}(u)}^{*} M,\|z\|=1$, such that

(a) There exist smooth functions $\alpha_{i j}$ defined in an open neighborhood of $\gamma_{u}([0,1])$ such that

$$
\forall j=1, \cdots, m \sum_{i=m+1}^{r} u_{i}(t) \alpha_{i j}\left(\gamma_{u}(t)\right) \varphi_{i, z}\left(\gamma_{u}(t)\right)=0 ;
$$

(b) the PSF $\varphi_{i, z}, i=m+1, \cdots, r$, are solution of a first order linear differential system $(D S)_{z}^{\prime}$ of dimension $r-m$ with terminal condition $z$.

The analysis of the previous system of equations provides useful informations on the sets $S$ and $\mathcal{E}(S)$, and, in some cases, leads to their explicit characterization (see, for instance, $[5,7,8]$ ). It has to be pointed out that there does not exist, to the best of the author's knowledge, any interesting general result regarding the topological nature of $\mathcal{E}(S)$. In particular, it is not known if Sard's theorem holds for $\mathcal{E}$, i.e., if $\mathcal{E}(S)$ has measure zero.

Fix $q \notin \mathcal{E}(S)$. It remains to find a control $u_{0}$ such that $u_{0} \notin S$ and a $C^{1} \operatorname{arc} \pi:[0,1] \rightarrow M$ such that $\pi(0)=\mathcal{E}\left(u_{0}\right)=p, \pi(1)=q, \pi([0,1]) \cap \overline{\mathcal{E}(S)}=\emptyset$. For instance, this can be done when $M \backslash \overline{\mathcal{E}(S)}$ is connected.

Step 2. By using (3), lift the path $\pi$ to a path $\Pi$ in $\mathcal{H}$. Then the existence of the global solution of (3) with $\Pi(0)=u_{0}$ must be established.

The lift $\Pi$ we are looking for should verify $\mathcal{E}(\Pi(s))=\pi(s)$ for $s \in[0,1]$ and $\Pi(0)=u_{0}$, i.e.

$$
D \mathcal{E}(\Pi(s)) \cdot \frac{\mathrm{d} \Pi}{\mathrm{d} s}(s)=\frac{\mathrm{d} \pi}{\mathrm{d} s}(s), \quad \text { a.e. in }[0,1], \Pi(0)=u_{0} .
$$

Since $\pi(s) \notin \mathcal{E}(S)$ for all $s \in[0,1]$, then $\operatorname{rank} D \mathcal{E}(\Pi(s))=n$. To solve (25), we use $P(u)$ the Moore-Penrose pseudo-inverse of $D \mathcal{E}(u)$ :

$$
\forall u \in \mathcal{H} \backslash S, P(u)=D \mathcal{E}(u)^{T_{\mathcal{H}}} G(u)^{-1}: T_{\mathcal{E}(u)} M \rightarrow \mathcal{H}, \text { and }\|P(u)\|=\left[\inf _{\|z\|=1}\langle z, G(u) z\rangle\right]^{-1 / 2} .
$$

We choose to take $\frac{\mathrm{d} \Pi}{\mathrm{d} s}(s)$ equal to

$$
\frac{\mathrm{d} \Pi}{\mathrm{d} s}(s)=P(\Pi(s)) \frac{\mathrm{d} \pi}{\mathrm{d} s}(s), \text { a.e. in }[0,1], \Pi(0)=u_{0},
$$

and in this way, (25) is verified. Equation (27) is the Path Lifting Equation (PLE). Recall that the HCM requires global existence of the solution of the PLE. The strategy we follow consists of proving, for certain controls $u$, a linear growth of $\|P(u)\|$ in terms of $\|u\|$. More precisely, let $K$ be a closed subset of $M$ such that $d(K, \overline{\mathcal{E}(S)})=\alpha>0$ and $\pi([0,1]) \subset K$. Assume that there exists $C_{K}>0$ such that, for every $u \in \mathcal{H}$ with $\mathcal{E}(u) \in K$, then $\|P(u)\| \leq C_{K}\|u\|_{\mathcal{H}}$, i.e., after using (15) and (26),

$$
\left(\exists C_{K}>0\right)(\forall u \in \mathcal{H})\left(\forall z \in T_{\mathcal{E}(u)}^{*} M,\|z\|=1\right)\left(\mathcal{E}(u) \in K \Rightarrow\|u\|_{\mathcal{H}}\left\|D \mathcal{E}(u)^{T_{\mathcal{H}}} z\right\|_{\mathcal{H}} \geq \frac{1}{C_{K}}\right) .
$$

Then, by Gronwall's lemma, the maximal solution of (27) is global i.e. defined on $[0,1]$. In the sequel, the strategy to establish the existence of a global solution will consists in proving (28).

The application of the HCM to the MPP can be summarized as follows: we first identify a subset $S$ of $\mathcal{H}$, the singular set, and its image $\mathcal{E}(S)$ on $M$. Next, we try to lift $C^{1}$ paths lying in the connected components of $M \backslash \overline{\mathcal{E}(S)}$ by solving the PLE, that is an o.d.e. in $\mathcal{H}$. That last step is reduced to establish Statement (28) for appropriate paths $\pi$. 
Remark 3. It is important to stress on the explicit character of a (possible) solution provided by the HCM to the MPP. Indeed, as regards existence results, there exist general ones. More precisely, if the map $\mathcal{E}$ is associated to a CC-tempered control system, then $\mathcal{E}$ satisfies the path lifting property (PLP), i.e., for every continuous path $\pi:[0,1] \rightarrow M$ with $\pi(0)=\mathcal{E}\left(u_{0}\right), u_{0} \in \mathcal{H}$, there exists a continuous path $\Pi_{c}:[0,1] \rightarrow \mathcal{H}$ satisfying $\mathcal{E} \circ \Pi=\pi$. This actually follows directly from the LARC (see [11] for instance). Note that the preceding lift $\Pi_{c}$ does not satisfy equation (25) in general.

\subsection{General properties of the PLE}

Let $\Sigma=\left(M, \mathbb{R}^{m}, \mathcal{H}, f\right)$ be a CC-tempered control system and $\mathcal{E}$ be an end-point map associated to some fixed point $p$.

\subsubsection{Local existence and uniqueness of the maximal solution of the PLE}

The Moore-Penrose inverse $P$ is Fréchet differentiable on $\mathcal{H} \backslash S$ since the end-point map $\mathcal{E}$ is actually smooth ( $c f$. Bismuth [3]). Using this fact, we get the next proposition.

Proposition 2. Let $\pi:[0,1] \rightarrow M \backslash \overline{\mathcal{E}(S)}$ be a $C^{1}$ curve. Then, for any couple $(\bar{s}, \bar{u})$ in $[0,1] \times \mathcal{H} \backslash S$, we have local existence and uniqueness of the maximal solution $\Pi$ of (27) with initial condition $\Pi(\bar{s})=\bar{u}$.

Proof of Proposition 2. Let $K=\pi([0,1])$ and $V$ an open set of $M$ containing $K$. In Section 4.3, we prove the existence of a time-varying vector field $\{h(s, \cdot)\}_{s \in[0,1]}$ such that

(i) $h$ is continuous on $[0,1] \times M$ and $h(s, \cdot)$ is smooth for every $s \in[0,1]$;

(ii) for every $x \in M / V, h(\cdot, x):[0,1] \rightarrow T_{x} M$ is identically equal to 0 ;

(iii) $\forall s$ in $[0,1], h(s, \pi(s))=\frac{\mathrm{d} \pi}{\mathrm{d} s}(s), \exists C>0, \forall x$ in $M,\|h(s, x)\| \leq\left\|\frac{\mathrm{d} \pi}{\mathrm{d} s}(s)\right\|$ and $\left\|D_{x} h(s, x)\right\| \leq C$.

Using $h$ defined above, we write the PLE (27):

$$
\frac{\mathrm{d} \Pi}{\mathrm{d} s}(s)=P(\Pi(s)) \cdot h(s, \mathcal{E}(\Pi(s))), \Pi(\bar{s})=\bar{u} .
$$

This o.d.e. is of the type $\frac{\mathrm{d} u}{\mathrm{~d} s}=F(s, u)$, with $F:[0,1] \times \mathcal{H} \rightarrow \mathcal{H}$ and $F(s, u)=P(u) \cdot h(s, \mathcal{E}(u))$. Since $P$ is Fréchet-differentiable in $\mathcal{H} \backslash S$ and $\frac{\mathrm{d} \pi}{\mathrm{d} s}$ is integrable in $[0,1]$, then $F$ is continuous, Fréchet-differentiable with respect to $u$, and there exists $C>0$ such that

$$
\forall u \in \mathcal{H} \backslash S, \forall s \in[0,1],\left\|D_{u} F(s, u)\right\| \leq C\left\|\frac{\mathrm{d} \pi}{\mathrm{d} s}(s)\right\| .
$$

The conditions of Carathéodory existence theorem hold, and hence, the proposition.

\subsubsection{A locally finite dimensional reduction of the PLE}

If $L$ is a closed linear subspace of $\mathcal{H}$, let $\operatorname{pr}_{L}$ be the orthogonal projector onto $L$ and $\mathcal{E}_{L}: L \rightarrow M$ the restriction of $\mathcal{E}$ to $L$. The image of $\mathcal{E}_{L}$ is equal to that of $\mathcal{E} \circ \operatorname{pr}_{L}: \mathcal{H} \rightarrow M$. For $u \in \mathcal{H}$, let $D \mathcal{E}_{L}(u): L \rightarrow T_{\mathcal{E}(u)} M$ be the restriction of $D \mathcal{E}(u)$ to $L$, (i.e. $D \mathcal{E}_{L}(u)=D \mathcal{E}(u) \circ \operatorname{pr}_{L}$ ) and $D \mathcal{E}_{L}^{T_{L}}(u)$ be its transpose map. Set $G_{L}(u)=D \mathcal{E}_{L}(u) \circ D \mathcal{E}_{L}^{T_{L}}(u)$, the controllability Gramian restricted to $L$ and, if $D \mathcal{E}_{L}(u)$ is onto, $P_{L}(u)$, the Moore-Penrose inverse of $D \mathcal{E}_{L}(u)$. Note that, if $u \in L$, then the differential of $\mathcal{E}_{L}$ at $u$, its transpose map, its controllability Gramian and the associated Moore-Penrose inverse coincide respectively with $D \mathcal{E}_{L}(u), D \mathcal{E}_{L}^{T_{L}}(u)$, $G_{L}(u)$ and $P_{L}(u)$.

Since $D \mathcal{E}_{L}^{T_{L}}(u)=\operatorname{pr}_{L} \circ D \mathcal{E}(u)^{T}$, we have, for every $z \in T_{\mathcal{E}(u)}^{*} M$,

$$
\langle z, G(u) z\rangle=\left\langle z, G_{L}(u) z\right\rangle+\left\|\left(I d_{\mathcal{H}}-\operatorname{pr}_{L}\right) D \mathcal{E}(u)^{T} z\right\|^{2} .
$$


The above equation shows, in particular if $D \mathcal{E}_{L}(u)$ is onto (and thus if $D \mathcal{E}(u)$ is) that

$$
\|P(u)\| \leq\left\|P_{L}(u)\right\| .
$$

We prove the following theorem.

Theorem 1. Let $\pi:[0,1] \rightarrow M \backslash \overline{\mathcal{E}(S)}$ be a $C^{1}$-curve and $\left(\mathcal{H}^{j}\right)_{j \geq 1}$ be a strictly increasing sequence of finitedimensional subspaces of $\mathcal{H}$ such that $\bigcup_{j \geq 1} \mathcal{H}^{j}$ is dense in $\mathcal{H}$. Assume that there exists $u_{0} \in \mathcal{H}^{j_{0}}$ for some $j_{0}$ and $\mathcal{E}\left(u_{0}\right)=\pi(0)$. Let $I$ be the interval of existence of the maximal solution of the PLE starting at $u_{0}$. Then, for every closed subinterval $J=\left[0, s_{0}\right] \subset I$, there exists $j_{1}$ so that, for every $j \geq j_{1}, \pi_{J}$, the restriction of $\pi$ to $J$, can be lifted to a path $\Pi^{J}$ in $\mathcal{H}^{j}$ verifying

(a) for every $s \in J, D \mathcal{E}_{\mathcal{H}^{j}}\left(\Pi^{J}(s)\right)$ is onto;

(b) the path $\Pi^{J}$ is the global solution on $J$ of the ODE in $\mathcal{H}^{j}$ given by

$$
\left\{\begin{array}{l}
\frac{\mathrm{d} \Pi^{J}}{\mathrm{~d} s}(s)=P_{\mathcal{H}^{j}}\left(\Pi^{J}(s)\right) \frac{\mathrm{d} \pi_{J}(s)}{\mathrm{d} s}, \\
\Pi^{J}(0)=u_{0} .
\end{array}\right.
$$

Equation (32) is, in a sense, a finite-dimensional version in $\mathcal{H}^{j}$ of the PLE in $\mathcal{H}$. In particular, if the PLE has a global solution, then $J$ can be taken equal to $[0,1]$. This result is similar in spirit to the finite-dimensional reduction of a general alternative problem (cf. [9] for a definition of an alternative problem and, [4,13] for finite-dimensional reduction procedures). Since $\mathcal{H}$ is a Hilbert space, such a finite-dimensional reduction of the PLE can be obtained by taking the span of a finite number of elements of a complete orthonormal system. Therefore, as noticed in [9], a result like Theorem 1 can be seen as a theoretical justification of the use of Galerkin's procedure in numerical implementations of the PLE.

Proof of Theorem 1. Consider a compact set $K \subset M \backslash \overline{\mathcal{E}(S)}$, containing $\pi(J)$ in its interior. For simplicity, we use $\operatorname{pr}_{j}, D \mathcal{E}_{j}, D \mathcal{E}_{j}^{T_{j}}, \ldots$ instead of $\operatorname{pr}_{\mathcal{H}^{j}}, D \mathcal{E}_{\mathcal{H}^{j}}, D \mathcal{E}_{\mathcal{H}^{j}}^{T_{\mathcal{H}^{j}}}, \ldots$, for $j \geq 1$.

For $\alpha>0$, let $\mathcal{K}_{\alpha}$ be the set of $u \in \mathcal{H}$ such that $\mathcal{E}(u) \in K$ and

$$
\min _{s \in[0,1]}\|u-\Pi(s)\|=\alpha .
$$

Clearly, for $\alpha>0$ is small enough, $\mathcal{K}_{\alpha}$ is a a closed set, containing $\Pi(J)$ in its interior. We first show the following lemma.

Lemma 2. There exist $\alpha_{0}, C_{0}>0$ and $j_{1}$ such that, for every $j \geq j_{1}$,

(a) $\max _{s \in J}\left\|\left(I d_{\mathcal{H}}-\operatorname{pr}_{j}\right) \circ D \mathcal{E}(\Pi(s))^{T}\right\| \rightarrow_{j \rightarrow \infty} 0$;

(b) for every $u \in \mathcal{K}_{\alpha_{0}},\left\|P_{j}(u)\right\| \leq 2\|P(u)\|$;

(c) $P_{j}$ is globally Lipschitz over $\mathcal{K}_{\alpha_{0}}$ with constant $C_{0}$.

Proof of Lemma 2. Item (a) follows from the fact that the subset of $\mathcal{H}$

$$
\left\{D \mathcal{E}^{T}(\Pi(s)) z, \quad s \in[0,1], z \in T_{\pi(s)}^{*} M,\|z\|=1\right\},
$$

is compact and the definition of the sequence $\left(\mathcal{H}^{j}\right)_{j \geq 1}$.

To establish items $(b)$ and $(c)$, it is enough to prove that there exist $\alpha_{0}>0$, and $j_{1} \geq 1$ such that for every $j \geq j_{1}$ and $u \in \mathcal{K}_{\alpha_{0}}$, we have, for every $z \in T_{\mathcal{E}(u)}^{*} M$ and $\|z\|=1$,

$$
\langle z, G(u) z\rangle \leq 2\left\langle z, G_{j}(u) z\right\rangle .
$$


Indeed, item $(b)$ directly follows from $(33)$ and for item $(c)$, one uses in addition the differentiation of

$$
P_{j}(u)=\operatorname{pr}_{j} D \mathcal{E}(u)^{T} G_{j}^{-1}(u) .
$$

In order to obtain (33), we start from (30) considered for $u$ in some $\mathcal{K}_{\alpha}\left(\alpha>0\right.$ small enough) and $z \in T_{\mathcal{E}(u)}^{*} M$, $\|z\|=1$. Let $s_{u} \in[0,1]$ such that $\left\|u-\Pi\left(s_{u}\right)\right\|=\alpha$. If $M$ were an Euclidean space, then $T_{\mathcal{E}(u)}^{*} M$ could be identified with $T_{\pi\left(s_{u}\right)}^{*} M$ and we would write at once $\left(I d_{\mathcal{H}}-\operatorname{pr}_{j}\right) D \mathcal{E}(u)^{T} z$ as the sum of two terms

$$
\left(I d_{\mathcal{H}}-\operatorname{pr}_{j}\right)\left[D \mathcal{E}(u)^{T}-D \mathcal{E}\left(s_{u}\right)^{T}\right] z+\left(I d_{\mathcal{H}}-\operatorname{pr}_{j}\right) D \mathcal{E}\left(s_{u}\right)^{T} z
$$

The first term is majorized by $C_{1} \alpha, C_{1}>0$ independent of $\alpha>0$ small enough, and, according to item ( $a$ ), the second term tends to zero as $j$ goes to infinity uniformly with respect to $u \in \mathcal{K}_{\alpha}$.

In the general situation, one can still show that

$$
\left\|\left(I d_{\mathcal{H}}-\operatorname{pr}_{j}\right) D \mathcal{E}(u)^{T} z\right\| \leq C_{1} \alpha+r_{j}
$$

where $C_{1}>0$ is independent of $\alpha>0$ small enough and $\lim _{j \rightarrow \infty} r_{j}=0$, uniformly with respect to $u \in \mathcal{K}_{\alpha}$. An argument goes as follows: consider the segment in $\mathcal{K}_{\alpha}$ defined by $v(\xi)=\Pi\left(s_{u}\right)+\xi\left(u-\Pi\left(s_{u}\right)\right)$ for $\xi \in[0,1]$. For $w \in T_{\pi\left(s_{u}\right)}^{*} M$, let $f_{w}$ be the map defined in a neighborhood $U$ of $\pi\left(s_{u}\right)$ that associates to $x \in U$ the cotangent vector obtained as the parallel transport of $w$ along the minimizing geodesic joining $\pi\left(s_{u}\right)$ and $x$. If $\alpha$ is small enough, there is a unique $z_{u} \in T_{\pi\left(s_{u}\right)}^{*} M$ such that $f_{z_{u}}(\mathcal{E}(u))=z$. Moreover that choice of $\alpha$ can be made uniform with respect to $u \in \mathcal{K}_{\alpha}$. It is also clear that $\frac{1}{2} \leq\left\|z_{u}\right\| \leq \frac{3}{2}$ for $\alpha$ small enough. Finally, for $\xi \in[0,1]$, define the differentiable mapping

$$
M(\xi)=\left(I d_{\mathcal{H}}-\operatorname{pr}_{j}\right) D \mathcal{E}(v(\xi))^{T} f_{z_{u}}(\mathcal{E}(v(\xi)))
$$

Of course $\left(I d_{\mathcal{H}}-\operatorname{pr}_{j}\right) D \mathcal{E}(u)^{T} z=M(1)$. Writing it as $M(0)+\int_{0}^{1} M^{\prime}(\xi) \mathrm{d} \xi,(34)$ follows.

Using (34), equation (30) becomes

$$
\left\langle z, G_{j}(u) z\right\rangle \geq\langle z, G(u) z\rangle-C_{1} \alpha-r_{j}
$$

where $C_{1}$ is independent of $\alpha, j \geq 1$ and $\lim _{j \rightarrow \infty} r_{j}=0$, uniformly with respect to $u \in \mathcal{K}_{\alpha}$.

Moreover $\langle z, G(u) z\rangle \geq \lambda_{\min }(G(u))$, the smallest eigenvalue of the positive definite symmetric matrix $G(u)$. We next show that there exists $\lambda^{0}>0$ independent of $\alpha$ small enough so that,

$$
\forall u \in \mathcal{K}_{\alpha}, \lambda_{\min }(G(u)) \geq \lambda^{0} .
$$

Arguing by contradiction, there is a sequence $\left(u^{k}\right)_{k \geq 1}$ of elements of $\mathcal{K}_{\alpha}$ such that $\lambda_{\min }\left(G\left(u^{k}\right)\right)$ tends to zero as $k$ goes to infinity. Up to a subsequence, $\left(u^{k}\right)_{k \geq 1}$ converges weakly to some $\bar{u} \in \mathcal{K}_{\alpha}$. This implies that $\left(\gamma_{u^{k}}\right)_{k \geq 1}$ converges to $\gamma_{\bar{u}}$ uniformly on $[0,1]$. Therefore, for every $z \in T_{\mathcal{E}(\bar{u})}^{*} M$ and sequence $\left(z^{k}\right)_{k \geq 1}$ with $z^{k} \in T_{\mathcal{E}\left(u^{k}\right)}^{*} M,\left\|z^{k}\right\|=1$ and $\lim _{k \rightarrow \infty} z_{k}=z$, then $D \mathcal{E}\left(u^{k}\right)^{T} z^{k}$ tends to $D \mathcal{E}(\bar{u})^{T} z$ as $k$ goes to infinity. Then $\lim _{k \rightarrow \infty} \lambda_{\min }\left(G\left(u^{k}\right)\right)=0$ implies that there exists $\bar{z} \in T_{\mathcal{E}(\bar{u})}^{*} M,\|\bar{z}\|=1$ such that $\langle\bar{z}, G(\bar{u}) \bar{z}\rangle=0$, i.e. $\bar{u}$ is an abnormal control. This contradicts the fact that $\mathcal{E}(\bar{u}) \in K \subset M \backslash \overline{\mathcal{E}(S)}$.

Using (35), we can conclude the proof of Lemma 2. By choosing $\alpha$ small enough and $j_{1}$ large enough, one has, for every $u \in \mathcal{K}_{\alpha}$ and $j \geq j_{1}, C_{1} \alpha+r_{l} \leq \frac{\lambda^{0}}{2}$. Therefore, (33) follows.

We now finish the proof of Theorem 1.For $j \geq j_{1}$, we consider the Cauchy problem given by

$$
\left\{\begin{array}{l}
\frac{\mathrm{d} \Pi^{j}}{\mathrm{~d} s}(s)=P_{j}\left(\Pi^{j}(s)\right) \frac{\mathrm{d} \pi_{J}(s)}{\mathrm{d} s}, \\
\Pi^{j}(0)=u_{0} .
\end{array}\right.
$$


We must show that there exists $j_{1}$ large enough so that $J_{j_{1}}=J$, where $J_{j_{1}}$ is the maximal interval of existence of the solution $\Pi^{j_{1}}$ of (36). Let $j \geq j_{0}$. As long as $\Pi^{j}$ belongs to $\mathcal{K}_{\alpha_{0}}$, then it is defined. Since $\Pi^{j}(0)=u_{0}$, there exists a nonempty time interval $[0, a)$ included in $J_{j}$ so that $\Pi^{j}$ is in the interior of $\mathcal{K}_{\alpha_{0}}$. For $s \in[0, a)$, set $v_{j}(s)=\Pi^{j}(s)-\Pi(s)$. Then $v_{j}(0)=0$ and

$$
\frac{\mathrm{d} v_{j}}{\mathrm{~d} s}(s)=\left[P_{j}\left(\Pi^{j}(s)\right)-P_{j}(\Pi(s))\right] \frac{\mathrm{d} \pi_{J}(s)}{\mathrm{d} s}+g_{j}(s),
$$

where $g_{j}(s)=\left[P_{j}(\Pi(s))-P(\Pi(s))\right] \frac{\mathrm{d} \pi_{J}(s)}{\mathrm{d} s}$. Note that $g_{j}$ is actually defined on the whole interval $J$. As for item (a) in Lemma 2, one has that $\lim _{j \rightarrow \infty} R_{j}=0$, where $R_{j}=\sup _{s \in J}\left|g_{j}(s)\right|$. Using item $(c)$ and applying Gronwall's lemma, one gets that

$$
\left\|v_{j}(s)\right\| \leq R_{j} \frac{\mathrm{e}^{C_{0} s}-1}{C_{0}}
$$

as long as the the right-hand side of the above inequality remains smaller than $\alpha_{0}$. Finally, by choosing $j$ large enough, that right-hand side stays smaller than $\alpha_{0}$ for every $s \in[0,1]$ and we conclude.

\subsubsection{A regularity theorem for the maximal solution of the PLE}

Let us consider a CC-tempered control system $\Sigma=\left(M, \mathbb{R}^{m}, \mathcal{H}, f\right)$ such that $\mathcal{H}=L^{2}\left([0,1], \mathbb{R}^{m}\right)$. Pick a curve $\pi:[0,1] \rightarrow M \backslash \overline{\mathcal{E}(S)}$ such that $\pi(0)=x_{1} \in M$ and $\pi(1)=q \in M$. For simplicity, we assume that $\pi$ is smooth. Let $u_{0}$ be the initial condition of the PLE associated to $\pi$. (In particular, $\mathcal{E}\left(u_{0}\right)=x_{1}$.) We only know a priori that $\Pi$, the maximal solution of the PLE, belongs to $\mathcal{H}$. It turns out that if $u_{0}$ is chosen more regular, then this regularity will be preserved as long as $\Pi$ is defined. More precisely, the following theorem holds.

Theorem 2. Let $\pi:[0,1] \rightarrow M \backslash \overline{\mathcal{E}(S)}$ be a smooth curve such that $\pi(0)=x_{1} \in M$ and $\pi(1)=q \in M$.

(a) Assume that $\mathcal{H}=L^{2}\left([0,1], \mathbb{R}^{m}\right)$ and consider an element $u_{0}$ of $\mathcal{H}$, which is not an abnormal control and such that $u_{0} \in H^{k}\left([0,1], \mathbb{R}^{m}\right)$ for some integer $k \geq 0$ and $\mathcal{E}\left(u_{0}\right)=x_{1}$. Let $I$ be the maximal interval of existence of the solution of the PLE associated to $\pi$ and starting at $u_{0}$. Then, $\forall s \in I, \Pi(s)$ can be written as $u_{0}+M(s)$, where $M(s) \in H^{k+1}\left([0,1], \mathbb{R}^{m}\right)$.

(b) The same conclusion holds, when we replace $H^{k}$ with $C^{k}$ in $(a)$.

In particular, if $u_{0}$ is smooth and the PLE has a global solution, then the input solving the MPP (i.e. steering $x_{0}$ to $q$ ) is also smooth.

Proof of Theorem 2. We will consider several positive constants denoted $C_{i}$ that will depend only on $k$, some compact set $K$ and integer $r$, both defined below. In addition, the next lemma is used repeatedly.

Lemma 3 (cf. Adams [1]). Let $l$ be a positive integer. Then, there exists $C>0$ such that for all $u, v \in H^{l}(0,1)$, we have $u v \in H^{l}(0,1)$ and $\|u v\|_{H^{l}} \leq C\|u\|_{H^{l}}\|v\|_{H^{l}}$.

Without loss of generality, we may assume that $I=[0,1]$. For $s \in[0,1]$, we write

$$
\Pi(s)=u_{0}+M(s),
$$

where we define $M(s)$ as

$$
M(s) \stackrel{\text { def }}{=} \int_{0}^{s} \frac{\mathrm{d} \Pi}{\mathrm{d} \eta}(\eta) \mathrm{d} \eta=\int_{0}^{s} P(\Pi(\eta)) \frac{\mathrm{d} \pi}{\mathrm{d} \eta}(\eta) \mathrm{d} \eta
$$

First, note that there exists $C_{1}>0$ such that

$$
\forall s \in[0,1], \quad\|\Pi(s)\|_{L^{2}} \leq C_{1} .
$$

Next, recall that the solutions of (5) are continuous with respect to the input $u \in \mathcal{H}$, and then, thanks to (39), there exists a compact set $K$ of $M$ such that

$$
\forall s, t \in[0,1], \quad \gamma_{\Pi(s)}(t) \in K .
$$


Therefore, if $i=1, \cdots, m$, and $s, t \in[0,1]^{2}$, the vector fields $f_{i}$ 's and their differentials (up to order $2 k$ ) remain bounded by a positive constant $C_{2}$, when they are evaluated at $\gamma_{\Pi(s)}(t)$. Furthermore, the corresponding adjoint vectors with initial condition $z$ of norm one are also bounded by a positive constant $C_{3}$.

As a consequence of Proposition 1, the switching functions together with finitely many pseudo-switching functions satisfy a first order linear differential system of dimension $r$ (independent of $s \in[0,1]$ ). Since $G^{-1}$ is continuous, there exists a positive constant $C_{4}$, such that

$$
\forall s \in[0,1], \quad\left\|G^{-1}(\Pi(s))\left(\frac{\mathrm{d} \pi}{\mathrm{d} s}(s)\right)\right\| \leq C_{4} .
$$

By definition of the Moore-Penrose inverse, we have, for $\eta \in[0,1]$,

$$
P(\Pi(\eta)) \frac{\mathrm{d} \pi}{\mathrm{d} \eta}(\eta)=D \mathcal{E}(\Pi(\eta))^{T} G^{-1}(\Pi(\eta)) \frac{\mathrm{d} \pi}{\mathrm{d} \eta}(\eta) .
$$

Thanks to (13) and recalling the definition of the switching vector given in (12), we have

$$
P(\Pi(\eta)) \frac{\mathrm{d} \pi}{\mathrm{d} \eta}(\eta)=\left\|G^{-1}(\Pi(\eta)) \frac{\mathrm{d} \pi}{\mathrm{d} \eta}\right\| \varphi_{\eta, z_{\eta}},
$$

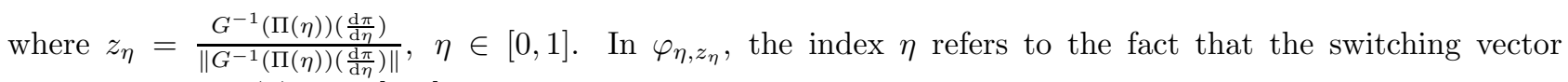
corresponds to $\Pi(\eta), \eta \in[0,1]$, and the index $z_{\eta}$ refers to the fact that the terminal condition of the adjoint equation along $\gamma_{\Pi(\eta)}$ is equal to $z_{\eta}$. We therefore write (38) as

$$
M(s)=\int_{0}^{s}\left\|G^{-1}(\Pi(\eta)) \frac{\mathrm{d} \pi}{\mathrm{d} \eta}\right\| \varphi_{\eta, z_{\eta}} \mathrm{d} \eta,
$$

Theorem 2 will be a consequence of the following lemma:

Lemma 4. Let $l \leq 2 k$ be an integer.

(i) Assume that,

(1i) for every $s \in[0,1], \Pi(s) \in H^{l}$;

(2i) there exists a positive constant $C_{5}$ such that

$$
\forall s \in[0,1], \quad\|\Pi(s)\|_{H^{l}} \leq C_{5} .
$$

Then there exists a positive constant $C_{6}$ such that,

$$
\forall s \in[0,1], \quad \forall z \in T_{\pi(s)}^{*} M,\|z\|=1, \quad \varphi_{s, z} \in H^{l+1} \text { and }\left\|\varphi_{s, z}\right\|_{H^{l+1}} \leq C_{6} .
$$

In addition, we have that, for every $s \in[0,1], M(s) \in H^{l+1}$.

(ii) Assume that

(1ii) for every $s \in[0,1], \Pi(s) \in C^{l}$;

(2ii) there exists a positive constant $C_{7}$, such that

$$
\forall s \in[0,1], \quad\left\|\Pi(s)^{(l)}\right\|_{L^{\infty}} \leq C_{7} .
$$

Then there exists a positive constant $C_{8}$ such that,

$$
\forall s \in[0,1], \quad \forall z \in T_{\pi(s)}^{*} M,\|z\|=1, \quad\left\|\varphi_{s, z}^{(l+1)}\right\|_{L^{\infty}} \leq C_{8} .
$$


Moreover,

$$
\forall s \in[0,1], \quad M(s) \in C^{l+1} .
$$

We first finish the proof of Theorem 2 assuming Lemma 4, and then provide its proof.

The conclusion of the theorem is shown by an inductive argument. For $k=0$, we already know that for every $s \in[0,1], \Pi(s) \in \mathcal{H}$. Then the conditions of Lemma 4 part $(i)$ are satisfied, and hence, we get Theorem 2 part $(a)$ for $k=0$. In particular, for every $s \in[0,1], M(s)$ is continuous. Therefore, if $u_{0}$ is also continuous, we have that for every $s \in[0,1], \Pi(s)$ is continuous and, clearly, $\|\Pi(s)\|_{L^{\infty}}$ is bounded by some positive constant independent of $s \in[0,1]$. Hence, by Lemma $4, M(s)$ is continuous and Theorem 2 part $(b)$ is proven for $k=0$.

Assume now that Theorem 2 holds for all the integers $l \in\{0, \cdots, k\}$. Consider $u_{0} \in H^{k+1}$. Then, by applying Theorem 2 to $u_{0} \in H^{k}, M(s) \in H^{k+1}$ for every $s \in[0,1]$. It implies that

$$
\forall s \in[0,1], \quad \Pi(s)=u_{0}+M(s) \in H^{k+1} .
$$

The conditions of Lemma 4 part $(i)$ are satisfied with $l=k+1$ and then Theorem 2 part $(a)$ follows. Finally, consider $u_{0} \in C^{k+1}$. Then $M(s) \in C^{k+1}$ for every $s \in[0,1]$ and, as in $(48), \Pi(s) \in C^{k+1}$. Using Lemma 4 part $(i i)$, we can complete the proof of the induction step for $l=k+1$. Then, Theorem 2 part $(b)$ is proved.

Proof of Lemma 4. Thanks to (19), there exists an integer $r \geq m$ and a finite subset $\left\{f_{1}, \cdots, f_{m}, f_{m+1}, \cdots f_{r}\right\}$ of $L_{\Sigma}$, with $f_{m+1}, \ldots, f_{r}$ iterated Lie brackets of the $f_{i}$ 's, such that for every $x \in K$,

$$
\operatorname{span}\left(f_{1}(x), \ldots, f_{m}(x), f_{m+1}(x), \ldots, f_{r}(x)\right)=T_{x} M
$$

Let $\tilde{\varphi}_{s}, s \in[0,1]$, be the $r$-dimensional vector corresponding to the $\operatorname{SF} \varphi_{1}, \cdots, \varphi_{m}$ and suitable $\operatorname{PSF} \varphi_{m+1}, \cdots, \varphi_{r}$. By Proposition 1 and (22), we have

$$
\left\{\begin{array}{l}
\dot{\tilde{\varphi}}_{s}(t)=W_{s}(t) \tilde{\varphi}_{s}(t), \quad \text { a.e. in }[0,1] \\
\tilde{\varphi}_{s}(1)=\tilde{z}_{s}
\end{array}\right.
$$

where $W_{s}(t)=\left(w_{s, q, j}(t)\right)_{1 \leq q, j \leq r}$ and $\tilde{z}_{s}=\left(\left\langle z_{s}, f_{j}(\Pi(s))\right\rangle\right)_{1 \leq j \leq r}$.

Note that there exists a positive constant $C_{9}$, such that

$$
\forall s \in[0,1], \sup _{1 \leq q, j \leq r}\left\|w_{s, q, j}\right\|_{H^{l}} \leq C_{9} \sup \left(1,\|\Pi(s)\|_{H^{l}}^{l+1}\right) \leq\left(C_{9}, C_{5}^{l+1}\right)
$$

In addition, it is easy to see that there exists a positive constant $C_{10}$ such that

$$
\forall s \in[0,1] \quad\left\|\tilde{z_{s}}\right\| \leq C_{10}
$$

Finally, by Gronwall's Lemma and Lemma 3, we obtain that $(M)$ has a global solution in $H^{l+1}$ and that there exists a positive constant $C_{11}$ such that

$$
\forall s \in[0,1] \quad\left\|\tilde{\varphi}_{s}\right\|_{H^{l+1}} \leq C_{11}
$$

Taking (52) into account, we have that $M(s), s \in[0,1]$, admits a measurable $(l+1)$ derivative, namely

$$
M(s)^{(l+1)}(t)=\int_{0}^{s}\left\|G^{-1}(\Pi(\eta))\left(\frac{\mathrm{d} \pi}{\mathrm{d} \eta}\right)\right\| \varphi_{\eta, z_{\eta}}^{(l+1)}(t) \mathrm{d} \eta .
$$


Furthermore, using the Cauchy-Schwarz inequality and (53), we obtain

$$
\begin{aligned}
\|M(s)\|_{H^{l+1}}^{2} & =\sum_{j=0}^{j=l+1}\left\|M(s)^{(j)}\right\|_{L^{2}}^{2}=\sum_{j=0}^{j=l+1} \int_{0}^{1}\left(\int_{0}^{s}\left\|G^{-1}(\Pi(\eta))\left(\frac{\mathrm{d} \pi}{\mathrm{d} \eta}\right)\right\| \varphi_{\eta, z_{\eta}}^{(j)}(t) \mathrm{d} \eta\right)^{2} \mathrm{~d} t \\
& \leq \sum_{j=0}^{j=l+1} \int_{0}^{1} C_{4}^{2}\left(\int_{0}^{s}\left(\varphi_{\eta, z_{\eta}}^{(j)}(t)\right)^{2} \mathrm{~d} \eta\right) \mathrm{d} t \leq C_{4}^{2} \int_{0}^{s}\left\|\varphi_{\eta, z_{\eta}}\right\|_{H^{l+1}}^{2} \mathrm{~d} \eta \leq C_{5}^{2} C_{11}^{2} .
\end{aligned}
$$

Therefore, Lemma 4 part $(i)$ is established.

In order to show Lemma 4 part $(i i)$, we need to prove that $M(s)^{(l+1)}$, given by (53), is continuous. This follows from the Lebesgue dominated convergence theorem since $\tilde{\varphi}_{s}^{(l+1)}, s \in[0,1]$, is continuous and $\sup _{s \in[0,1]}\left\|\tilde{\varphi}_{s}^{(l+1)}\right\|_{L^{\infty}}<\infty$.

\section{The strong Bracket generating Case}

For the rest of the section, $n>m \geq 2$ and $\Sigma=\left(M^{n}, \mathbb{R}^{m}, \mathcal{H}_{m}, \bar{f}\right)$ is a CC-tempered control system satisfying the SBGC.

Consider on $T^{*} M$ the Hamiltonian lift $T^{*} \Sigma$ defined in (7). Set $F_{i}=T^{*} f_{i}$, for $i=1, \ldots, m$. Then, for $\xi=(x, z) \in T^{*} M$ and $i, j, k=1, \cdots, m$, we set

$$
\begin{gathered}
\varphi_{i}(\xi) \stackrel{\text { def }}{=}\left\langle z, f_{i}(x)\right\rangle, \varphi_{i, j}(\xi) \stackrel{\text { def }}{=}\left\langle z,\left[f_{j}, f_{i}\right](x)\right\rangle, \varphi_{i, j, k}(\xi) \stackrel{\text { def }}{=}\left\langle z,\left[f_{k},\left[f_{j}, f_{i}\right]\right](x)\right\rangle, \\
\varphi(\xi) \stackrel{\text { def }}{=}\left(\varphi_{1}(\xi), \cdots, \varphi_{m}(\xi)\right),\|\varphi(\xi)\| \stackrel{\text { def }}{=}\left(\sum_{i=1}^{m} \varphi_{i}(\xi)^{2}\right)^{1 / 2}, \mathcal{A}(\xi) \stackrel{\text { def }}{=}\left(\varphi_{i, j}(\xi)\right)_{i, j=1, \cdots, m} .
\end{gathered}
$$

Given a trajectory $\xi$ of $T^{*} \Sigma$, let $\varphi_{i}(t), \varphi_{i, j}(t), \varphi_{i, j, k}(t), \varphi(t)$ and $\mathcal{A}(t)$ simply denote $\varphi_{i}(\xi(t)), \varphi_{i, j}(\xi(t))$, $\varphi_{i, j, k}(\xi(t)), \varphi(\xi(t))$ and $\mathcal{A}(\xi(t))$ respectively. We recall that

$$
\varphi^{\prime}(t)=\mathcal{A}(t) u(t)
$$

We now give two consequences of the SBGC in terms of the switching functions.

Proposition 3. Let $\Sigma$ be a driftless control-affine system on $M$ subject to the $S B G C$. For $\xi=(x, z) \in T^{\sharp} M$, assume that $\varphi_{1}(\xi)=\cdots=\varphi_{m}(\xi)=0$. Then the following two properties hold and are equivalent:

(a) the $F$-Lie derivatives of the $S F \varphi_{i},\left(\nabla_{F} \varphi_{i}(\xi)\right)_{i=1, \ldots, m}, \operatorname{span} \mathbb{R}^{m}$.

(b) $\mathcal{A}(\xi)$ is invertible.

When $\mathcal{A}(\xi)$ is invertible, set $\mathcal{A}^{-1}(\xi) \stackrel{\text { def }}{=}\left(b_{i j}(\xi)\right)_{1 \leq i, j \leq m}$.

Proof of Proposition 3. We write

$$
\begin{aligned}
F_{j} \cdot \varphi_{i}(\xi) & =\mathrm{d} \varphi_{i} \cdot F_{j}=\left(\left\langle z, D f_{i}(x)(\cdot)\right\rangle, f_{i}(x)\right)\left(\begin{array}{c}
f_{j}(x) \\
-\left\langle z, D f_{j}(x)(\cdot)\right\rangle
\end{array}\right) \\
& =\left\langle z, D f_{i}(x) \cdot f_{j}(x)-D f_{j}(x) f_{i}(x)\right\rangle=\left\langle z,\left[f_{j}, f_{i}\right](x)\right\rangle=\varphi_{i, j}(\xi)
\end{aligned}
$$

For $\theta=\left(\theta_{1}, \ldots, \theta_{m}\right)$ and $j=1, \ldots, m$, we have

$$
\sum_{i=1}^{m} \theta_{i} \nabla_{F} \varphi_{i}=-\left(\left\langle z,\left[\theta \cdot f, f_{j}\right](x)\right\rangle\right)_{1 \leq j \leq m}=-\mathcal{A}(\xi)(\theta)
$$


Hence, $\mathcal{A}(\xi) \theta=0$ is equivalent to

$$
\sum_{i=1}^{m} \theta_{i} \nabla_{F} \varphi_{i}=0
$$

We pick $\xi \in T^{\sharp} M$ with $\varphi(\xi)=0$. If $\left(\nabla_{F} \varphi_{i}(\xi)\right)_{i=1, \ldots, m}$ do not span $\mathbb{R}^{m}$, then, thanks to (55), there exists $\bar{\theta} \neq 0$ such that $\left\langle z, f_{i}(x)\right\rangle=0$ and $\left\langle z,\left[\bar{\theta} \cdot f, f_{i}\right](x)\right\rangle=0$, for $1 \leq i \leq m$. Therefore, there exists a nonzero vector $z \in T_{x} M$ perpendicular to the subspace $F$ of $T_{x} M$ generated by the $f_{i}(x)$ 's and the $\left[\bar{\theta} \cdot f, f_{i}\right](x)$ 's, $1 \leq i \leq m$. That contradicts the SBGC. Hence, the proposition follows.

\subsection{Case where $\mathcal{H}=L^{2}\left([0,1], \mathbb{R}^{m}\right)$}

Consequence $(b)$ of the SBGC was used by Sussmann in [31] to prove the following theorem:

Theorem 3. Let $\Sigma=\left(M, \mathbb{R}^{m}, \mathcal{H}, f\right)$ be a CC-tempered control system where $\mathcal{H}=L^{2}\left([0,1], \mathbb{R}^{m}\right), f$ is an $m$-tuple of $C^{3}$ vector fields and the $S B G C$ holds. Let $x_{0} \in M$ and $\mathcal{E}$ the corresponding end-point map. Then every $C^{1}$ path $\pi$ such that $x_{0} \notin \pi([0,1])$ can be lifted through $\mathcal{E}$ using the $H C M$.

Here we describe another proof of this theorem (as given in [8]), based on consequence $(a)$ of the SBGC. The general structure of the proof is that of [31] and it will be provided next because that structure of proof will be the one for the argument of Theorem 4.

It consists in applying Proposition 4 in several cases.

Proof of Theorem 3. Let $x_{0} \in M$ and consider the corresponding end-point map $\mathcal{E}: \mathcal{H} \rightarrow M$. Since the control system satisfies the SBGC, it follows from Proposition $3(b)$ that the singular set $S$ reduces to $\{0\}$ (see also Strichartz [30]). Hence, $\mathcal{E}(S)=\left\{x_{0}\right\}$. Let $\pi$ be a $C^{1}$ path such that $x_{0} \notin \pi([0,1])$. Consider $u_{0} \in \mathcal{H}$ with $\mathcal{E}\left(u_{0}\right)=\pi(0)$. Such a control exists since $\Sigma$ is completely controllable and $\mathcal{E}(S)=\left\{x_{0}\right\}$. We must show that the PLE corresponding to $\pi$ with initial condition $u_{0}$ admits a global solution on $[0,1]$.

Let $d$ ( $d^{*}$ respectively) be the distance function induced by the given complete Riemannian metric on $M$ ( $T^{*} M$ respectively). For $\alpha>0$ and $A$ a bounded set in a finite dimensional manifold, we define the following compact set

$$
K_{\alpha}(A) \stackrel{\text { def }}{=}\left\{y \in M \mid \inf _{s \in A} d(s, y) \leq \alpha\right\} .
$$

Let $K_{\alpha_{0}}=K_{\alpha_{0}}(\pi([0,1]))$, where $\alpha_{0}$ is chosen small enough so that $x_{0} \notin K_{\alpha_{0}}$. We define the compact subsets $J$ and $Z_{J}$ of $T^{\sharp} M$ by

$$
\begin{aligned}
J & =\left\{\xi=(x, z) \mid x \in K_{\alpha_{0}}, z \in T_{x_{0}}^{*} M, \frac{1}{2} \leq\|z\| \leq \frac{3}{2}\right\} \\
Z_{J} & =\left\{\xi \in J \mid \varphi_{1}(\xi)=\cdots=\varphi_{m}(\xi)=0\right\} .
\end{aligned}
$$

Therefore, by Proposition $3, \min _{\xi \in Z_{J}}\left|\operatorname{det}\left(\nabla_{F} \varphi_{j}\right)_{j=1, \ldots, m}\right|>0$. Let $\mathcal{A}_{J, \varphi}$ be equal to $K_{\tilde{\alpha}}\left(Z_{J}\right)$ for some $\tilde{\alpha}>0$, such that

$$
\min _{\xi \in \mathcal{A}_{J, \varphi}}\left|\operatorname{det}\left(\nabla_{F} \varphi_{j}(\xi)\right)_{j=1, \ldots, m}\right|>0
$$

A crucial step in the proof of Theorem 3 relies on Proposition 4 (see below), which is of independent interest. Its proof is not given since it is a simpler version of the main argument of [8].

Proposition 4. Let $\Sigma$ be the control system $\left(M, \mathbb{R}^{m}, \mathcal{H}, f\right)$ where $\mathcal{H}=L^{2}\left((0,1), \mathbb{R}^{m}\right)$ and $f$ is an m-tuple of $C^{1}$ vector fields. Let $\varphi_{1}, \ldots, \varphi_{r}, r \geq m: T^{*} M \rightarrow \mathbb{R}$ be $C^{2}$ functions and set $\varphi=\left(\varphi_{1}, \ldots, \varphi_{r}\right)$.

For a compact set $K \subseteq M$, consider the sets $J(K)$ and $Z(K)$ associated to $K$ as $J$ and $Z_{J}$ were associated to $K_{\alpha_{0}}$ in (57) and (58). Let $\psi: T^{*} M \rightarrow \mathbb{R}$ be a $C^{2}$ function satisfying

There exist a compact neighborhood $\mathcal{N}=\mathcal{N}_{\psi, J(K), \varphi}$ of $Z(K)$,

$C^{1}$ functions $\beta_{1}, \ldots, \beta_{r}: \mathcal{N} \rightarrow \mathbb{R}, C^{0} \mathbb{R}^{m}$-valued functions $\delta_{1}, \cdots, \delta_{r}: \mathcal{N} \rightarrow \mathbb{R}^{m}$, $\forall \xi \in \mathcal{N}, \nabla_{F} \psi(\xi)=\sum_{j=1}^{r}\left(\beta_{j}(\xi) \nabla_{F} \varphi_{j}(\xi)+\varphi_{j}(\xi) \delta_{j}(\xi)\right)$. 
Then, there exists $C=C_{J(K), \psi, \varphi}>0$ such that, for any subinterval $[a, b]$ in $[0,1]$ and any control $u \in$ $L^{2}\left([a, b], \mathbb{R}^{m}\right)$ that gives rise to a trajectory $\xi$ of $T^{*} \Sigma$ contained in $J(K)$, we have

$$
|\psi(\xi(b))-\psi(\xi(a))| \leq C\|u\|_{L^{2}([a, b])}\|\varphi\|_{L^{2}, \xi},
$$

where $\|\varphi\|_{L^{2}, \xi}^{2} \stackrel{\text { def }}{=} \sum_{j=1}^{r} \int_{a}^{b} \varphi_{j}(\xi(t))^{2} \mathrm{~d} t$ and $\|u\|_{L^{2}([a, b])}^{2} \stackrel{\text { def }}{=} \sum_{j=1}^{m} \int_{a}^{b} u_{j}^{2}(t) \mathrm{d} t$.

As a consequence of the SBGC, it is easy to show that every function $\psi: T^{\sharp} M \rightarrow \mathbb{R}$ of class $C^{2}$ satisfies Hypothesis $(\Upsilon)$ with $\varphi$ equal to the $m$-tuple of SF. Consider the following two functions $\psi_{1}, \psi_{2}: T^{\sharp} M \rightarrow \mathbb{R}$ of class $C^{\infty}$ defined as $\psi_{1}(x, z)=\|z\|$ and $\psi_{2}=\tilde{\psi}_{2} \circ \pi^{\sharp}$, where $\tilde{\psi}_{2}: M \rightarrow \mathbb{R}^{+}$is $C^{\infty}$ and satisfies

$$
\forall x \in M, 1 / 2 \leq \tilde{\psi}_{2}(x) \leq 1, \tilde{\psi}_{2}(x) \equiv 1 / 2 \text {, if } x \in K_{\alpha_{0} / 4}, \tilde{\psi}_{2}(x) \equiv 1 \text {, if } x \notin K_{\alpha_{0} / 2} .
$$

We apply Proposition 4 twice, first to $\varphi, J$ and $\psi_{1}$, and then to $\varphi, J$ and $\psi_{2}$. Here $J$ is the set defined in (57). We conclude the existence of $C>0$, such that for every subinterval $[a, b]$ of $[0,1]$ and for every $u \in L^{2}\left([a, b], \mathbb{R}^{m}\right)$ that gives rise to a trajectory $\xi$ contained in $J$, we have

$$
\sup _{i=1,2}\left|\psi_{i}(\xi(b))-\psi_{i}(\xi(a))\right| \leq C\|u\|_{L^{2}([a, b])}\|\varphi\|_{L^{2}, \xi}
$$

Take $u \in \mathcal{H}$ such that $\mathcal{E}(u) \in \pi([0,1])$ and consider the largest subinterval $[a, 1]$ of $[0,1]$ for which $\gamma_{u}([a, 1])$ is contained in $K_{\alpha_{0}}$. Since $x_{0}=\gamma_{u}(0) \notin K_{\alpha_{0}}, a>0$ and $\gamma_{u}(a) \in \partial K_{\alpha_{0}}$. Solving the adjoint equation (8) with the terminal condition $\lambda(1)=z,\|z\|=1$, we lift $\gamma_{u}:[a, 1] \rightarrow M$ to $\xi_{u, z}:[a, 1] \rightarrow T^{*} M$. Because $\xi_{u, z}(1)=\left(\gamma_{u}(1), z\right)$ belongs to $\stackrel{\circ}{J}$, there exists a maximal subinterval $\left[a^{\prime}, 1\right]$ of $[a, 1]$ such that $\xi_{u, z}\left(\left[a^{\prime}, 1\right]\right)$ is contained in $J$ and $\xi_{u, z}\left(a^{\prime}\right) \in \partial J$.

Two cases have to be considered: $a=a^{\prime}$ and $a<a^{\prime}$.

Case 1. $a=a^{\prime}$.

The whole lift $\xi_{u, z}$ is in $J$. Since $\gamma_{u}(a) \in \partial K_{\alpha_{0}}$, we have

$$
d\left(\gamma_{u}(a), \pi([0,1])=\alpha_{0}\right.
$$

Therefore, $\gamma_{u}(a)$ does not belong to $K_{\alpha_{0} / 2}$. Note that $\gamma_{u}(1)$ belongs to $K_{\alpha_{0} / 4}$. Then,

$$
\left|\psi_{1}\left(\xi_{u, z}(1)\right)-\psi_{1}\left(\xi_{u, z}(a)\right)\right|=\left|\frac{1}{2}-0\right|=\frac{1}{2} .
$$

Case 2. $a<a^{\prime}$.

We have $\xi_{u, z}\left(a^{\prime}\right) \in \partial J$, i.e. $\gamma_{u}\left(a^{\prime}\right) \in \partial K$ or $\left\|\lambda\left(a^{\prime}\right)\right\| \in\left\{\frac{1}{2}, \frac{3}{2}\right\}$.

The first alternative reduces to the previous case, while for the second one, we have

$$
\begin{aligned}
\left|\psi_{1}\left(\xi_{u, z}(1)\right)-\psi_{1}\left(\xi_{u, z}\left(a^{\prime}\right)\right)\right| & =\left|\|\lambda(1)\|-\left\|\lambda\left(a^{\prime}\right)\right\|\right| \\
& =\left|\|z\|-\left\|\lambda\left(a^{\prime}\right)\right\|=\right| 1-\frac{1}{2}|=| 1-\frac{3}{2} \mid=\frac{1}{2}
\end{aligned}
$$

Therefore, in both cases, we obtain from Proposition 4

$$
\frac{1}{2} \leq C\|u\|_{L^{2}\left(a^{\prime}, 1\right)}\|\varphi\|_{L^{2}, \xi_{u, z}}
$$

where $C$ is independent of $u \in \mathcal{H}$ with $\mathcal{E}(u) \in \pi([0,1])$ and $z \in T_{\mathcal{E}(u)}^{*} M$ as long as $\|z\|=1$. 
Using the notations introduced in Chapter 2, we have

$$
\|\varphi\|_{L^{2}, \xi_{u, z}}=\left\|\varphi_{z}\right\|_{L^{2}\left(a^{\prime}, 1\right)} \leq\left\|\varphi_{z}\right\|_{L^{2}(0,1)} .
$$

Let $C^{\prime}=\frac{1}{2 C}>0$. Then (61) implies that, for every $u \in \mathcal{H}$ for which $\mathcal{E}(u) \in \pi([0,1])$ and for every $z \in T_{\mathcal{E}(u)}^{*} M$ such that $\|z\|=1,\|u\|_{L^{2}(0,1)}\left\|\varphi_{z}\right\|_{L^{2}(0,1)} \geq C^{\prime}$. This completes the proof of Theorem 3 .

Following the strategy described in Section 2.3, the MPP can be solved, i.e., for any points $p, q \in M$, we can produce a control $u_{p, q}$ that steers $p$ to $q$. Pick two points $p, q \in M$ such that $p \neq q$. We consider the end-point map $\mathcal{E}: \mathcal{H} \rightarrow M$ corresponding to $p$. Since our control system satisfies the SBGC, $\mathcal{E}(S)=\{p\}$. We consider first $\bar{u}_{0} \not \equiv 0$ in $\mathcal{H}$. There exists a time $t_{1}$ in $[0,1]$ such that $\gamma_{\bar{u}_{0}}\left(t_{1}\right)=x_{0} \neq p$. We define the control $u_{0}$ by

$$
\begin{cases}\bar{u}_{0} & \text { on }\left[0, t_{1}\right] \\ 0 & \text { on }\left(t_{1}, 1\right]\end{cases}
$$

Thus, $\mathcal{E}\left(u_{0}\right)=x_{0} \neq p$. Since $n>1, \widetilde{M}=M \backslash p$ is arcwise connected, we can choose now a $C^{1}$ path $\pi$ in $\widetilde{M}$ joining $x_{0}$ to $q$. By Theorem $3, \pi$ can be lifted through $\mathcal{E}$ using the HCM to a path $\Pi$ and we take $u_{p, q}=\Pi(1)$.

\subsection{Other control spaces}

In this section, we are concerned with more regular Hilbert spaces defined next. For $r \geq 1$, the Hilbert space $H^{r, 1}$ was already introduced in Chapter 2 as an example of Hilbert space satisfying Property $(\mathrm{Cl})_{r}$. The spaces $H^{r, m}$ are the spaces of functions $u=\left(u_{1}, \cdots, u_{m}\right):[0,1] \rightarrow \mathbb{R}^{m}$, where each $u_{i} \in H^{r, 1}, i=1, \cdots, m$. These spaces are Hilbert spaces for the scalar products

$$
\forall u, v \in H^{r, m},\left\langle u, v>\stackrel{\text { def }}{=} \sum_{i=1}^{m} \int_{0}^{1} u_{i}^{(r)}(t) v_{i}^{(r)}(t) \mathrm{d} t .\right.
$$

The associated norms are denoted $\|\cdot\|$. We write, here and henceforth, the spaces $H^{r, m}$ simply $\mathcal{H}$. The main result of this section is the following:

Theorem 4. Let $\Sigma=\left(M, \mathbb{R}^{m}, \mathcal{H}, f\right)$ be a $C C$-tempered control system with $\mathcal{H}=H^{r, m}$ for some $r \geq 1$. Assume that $\Sigma$ satisfies the $S B G C$. Let $x_{0} \in M$ and $\mathcal{E}$ the corresponding end-point map. Then every $C^{1}$ path $\pi$ such that $x_{0} \notin \pi([0,1])$ can be lifted through $\mathcal{E}$ using the $H C M$.

Note that Theorem 4 has a natural corollary in the case of the MPP with obstacles.

Proof of Theorem 4. For $u \in \mathcal{H}, i=1, \cdots, m$, and $t \in[0,1]$, set

$$
U_{i}(t) \stackrel{\text { def }}{=} \int_{1}^{t} u_{i}(s) \mathrm{d} s, U(t) \stackrel{\text { def }}{=}\left(U_{1}(t), \cdots, U_{m}(t)\right), \bar{U}(t) \stackrel{\text { def }}{=} \sup _{[t, 1]} \sup _{i=1, \cdots, m}\left|U_{i}(s)\right| .
$$

Let $x_{0} \in M$ and let $\mathcal{E}: \mathcal{H} \rightarrow M, u \mapsto \gamma_{u}(1)$ be the corresponding end-point map. Under the SBGC, the set of abnormal controls reduces to $\{0\}$. Let $\pi:[0,1] \rightarrow M$ be a $C^{1}$ path and $u_{0} \in \mathcal{H}$ such that $\pi(0)=\mathcal{E}\left(u_{0}\right)$ and $x_{0} \notin \pi([0,1])$. We choose $\alpha>0$ small enough so that $K_{\alpha}(\pi([0,1]))$ is compact and $d_{M}\left(x_{0}, \pi([0,1])\right)>\alpha$. Consider $J$ and $Z(J)$ introduced in (57), (58) and define

$$
\mathcal{K} \stackrel{\text { def }}{=}\left\{\xi=(x, z) \in T^{*} M \mid x \in \pi([0,1]),\|z\|=1\right\} .
$$

Then choose $\beta>0$ small enough such that

$$
\min _{\xi \in K_{\beta}(Z(J))}|\operatorname{det} \mathcal{A}(\xi)|>0
$$


Introduce the following constants:

$$
\begin{gathered}
\rho_{1}=\min \left\{\|\varphi(\xi)\|, \xi \in \overline{\mathcal{K} / K_{\beta}(Z(J))}\right\}(>0), \\
D_{1}=\sup _{\substack{\xi \in J \\
1 \leq i, j, k \leq m}}\left(1,\|\varphi(\xi)\|,\left\|\varphi_{i, j}(\xi)\right\|,\left\|\varphi_{i, j, k}(\xi)\right\|,\|\mathcal{A}(\xi)\|,\|D \mathcal{A}(\xi)\|\right), \\
D_{2} \stackrel{\text { def }}{=} \sup _{J \cap K_{\beta}(Z(J))}\left(\left\|\mathcal{A}^{-1}(\xi)\right\|,\left\|D \mathcal{A}^{-1}(\xi)\right\|\right), D \stackrel{\text { def }}{=} \sup \left(D_{1}, D_{2}\right), \quad \bar{\rho} \stackrel{\text { def }}{=} \inf \left(\frac{\rho_{1}}{\sqrt{m}}, \frac{1}{24 m D}\right) .
\end{gathered}
$$

A direct computation shows that, for every $u \in \mathcal{H}$ and $z \in T_{\mathcal{E}(u)}^{*} M$, we have

$$
\langle z, G(u) z\rangle=\sum_{i=1}^{m}\left\|R_{i}\right\|_{H^{r, 1}}^{2}
$$

where, for each $i=1, \cdots, m, R_{i}$ is the $H^{r, 1}$ - representation of $\varphi_{i} \in L^{2}([0,1], \mathbb{R})$, i.e. we have

$$
\text { for } i=1, \cdots, m, \forall v \in H^{r, 1},\left\langle R_{i}, v\right\rangle=\int_{0}^{1} \varphi_{i}(s) v(s) \mathrm{d} s .
$$

For $i=1, \cdots, m$, we get that $(-1)^{r} R_{i}^{(2 r)}=\varphi_{i}$ with the boundary conditions

$$
R_{i}^{(j+r)}(0)=R_{i}^{(j)}(1)=0, j=0, \cdots, r-1 .
$$

Let $R=\left(R_{1}, \cdots, R_{m}\right)$. We rewrite $(62)$ as

$$
\langle z, G(u) z\rangle=\|R\|_{\mathcal{H}}^{2}=\left\|R^{(r)}\right\|_{L^{2}(0,1)}^{2} .
$$

Following the strategy of the proof of Theorem 3, the proof of Theorem 4 reduces to establish (28) which, by using (63), can be written

$$
(\exists C>0)(\forall u \in \mathcal{H})\left(\forall z \in T_{\mathcal{E}(u)}^{*}\right)\left((\mathcal{E}(u), z) \in \mathcal{K} \Rightarrow\left\|u^{(r)}\right\|_{L^{2}(0,1)}\left\|R^{(r)}\right\|_{L^{2}(0,1)} \geq C\right) .
$$

Let $u \in \mathcal{H}$ and $z \in T_{\mathcal{E}(u)}^{*} M$ with $(\mathcal{E}(u), z) \in \mathcal{K}$. If $\xi$ is the trajectory of $u$ associated to $T^{*} \Sigma$, then $\xi(1)=$ $\left(\gamma_{u}(1), z\right) \in \stackrel{\circ}{J}$. Therefore, there exists a maximal subinterval $\left[t_{0}, 1\right]$ of $[0,1]$ such that

$$
\text { (i) } t_{0}<1,(i i) \xi\left(\left[t_{0}, 1\right]\right) \subset \stackrel{\circ}{\complement},(i i i) \xi\left(t_{0}\right) \in \partial J
$$

Furthermore, we have

Lemma 5. There exists a subinterval $\left[t_{1}, 1\right]$ of $\left[t_{0}, 1\right]$, such that

$$
\left(P_{1}\right) \xi\left(\left(t_{1}, 1\right]\right) \subset \stackrel{\circ}{J}, \quad \text { and }\left(P_{2}\right) \forall t \in\left(t_{1}, 1\right], \bar{U}(t)<\frac{1}{6}
$$

Moreover, we have either

$\left(P_{3}\right)\|\varphi(1)\|<\frac{\rho}{2}, \forall t \in\left(t_{1}, 1\right]\|\varphi(t)\|<\rho$, and equality holds at $t=t_{1}$ for one $\left(P_{i}\right), i=1,2,3$,

or

$\left(P_{4}\right)\|\varphi(1)\| \geq \frac{\rho}{2}, \forall t \in\left(t_{1}, 1\right],\|\varphi(t)\|>\frac{\rho}{4}$, and equality holds at $t=t_{1}$ for one $\left(P_{i}\right), i=1,2,4$ 
Remark 4. Equality in $\left(P_{1}\right)$ says that $\xi\left(t_{1}\right) \in \partial J$.

Proof of Lemma 5. Let $t_{2}$ be such that $\bar{U}\left(t_{2}\right)=\frac{1}{6}$ and $\bar{U}(t)<\frac{1}{6}$ on $\left(t_{2}, 1\right]\left(\right.$ if $\bar{U}(0)<\frac{1}{6}$, we take $\left.t_{2} \stackrel{\text { def }}{=} 0\right)$. If $\|\varphi(1)\|<\rho / 2$, let $t_{4}$ be such that $\left\|\varphi\left(t_{4}\right)\right\|=\rho$ and $\forall t \in\left(t_{4}, 1\right],\|\varphi(t)\|<\rho\left(\right.$ if $\left.\forall t \in\left[t_{0}, 1\right],\|\varphi(t)\|<\rho, t_{4} \stackrel{\text { def }}{=} t_{0}\right)$. Set $t_{1} \stackrel{\text { def }}{=} \max \left(t_{2}, t_{4}, t_{0}\right)$.

Assume now that $\|\varphi(1)\| \geq \rho / 2$. Let $t_{3}$ be such that $\left\|\varphi\left(t_{3}\right)\right\|=\rho / 4$ and $\|\varphi(t)\|>\rho / 4$ on $\left(t_{3}, 1\right]($ if $\|\varphi(t)\|>\rho / 4$ on $\left.\left[t_{0}, 1\right], t_{3} \stackrel{\text { def }}{=} t_{0}\right)$. Set $t_{1} \stackrel{\text { def }}{=} \max \left(t_{2}, t_{3}, t_{0}\right)$. The statement of Lemma 5 follows.

We call the time $t_{1}$ obtained in Lemma 5 , the "first exit time" for the trajectory $\xi$ corresponding to the pair $(u, z)$ so that $(\mathcal{E}(u), z) \in \mathcal{K}$. We prove the following lemma.

Lemma 6. There exist $\rho \in(0, \bar{\rho}], \rho=\rho(\alpha, \bar{\rho}, D)$ and $C=C(\rho, \alpha, J)$, such that for every $u \in \mathcal{H}$ and $z \in T_{\mathcal{E}(u)}^{*} M$ so that $(\mathcal{E}(u), z) \in \mathcal{K}$, if $t_{1} \in[0,1)$ is the "first exit time" corresponding to $(u, z)$, then

$$
\|u\|_{L^{2}\left(\left[t_{1}, 1\right]\right)}\|\varphi\|_{L^{2}\left(\left[t_{1}, 1\right]\right)} \geq C
$$

Proof of Lemma 6. We have to consider several cases.

- Case 1. $\left(P_{3}\right)$ holds.

1.a. Equality in $\left(P_{1}\right)$ : The conclusion of the theorem follows since this subcase is treated Case 2 in the proof of Theorem 3 .

1.b. Equality in $\left(P_{2}\right)$ : In this case, there is an index $i_{1} \in(1, \cdots, m)$, such that

$$
\left|U_{i_{1}}\left(t_{1}\right)\right|=\frac{1}{6}
$$

By $\left(P_{3}\right), \mathcal{A}(t)$ is invertible for every $t \in\left[t_{1}, 1\right]$. Hence,

$$
\begin{aligned}
U_{i_{1}}\left(t_{1}\right) & =\int_{1}^{t_{1}} u_{i_{1}}(s) \mathrm{d} s=\int_{1}^{t_{1}}\left(\mathcal{A}^{-1} \varphi^{\prime}\right)_{i_{1}}(s) \mathrm{d} s=\int_{1}^{t_{1}} \sum_{j=1}^{m} b_{i_{1} j}(s) \varphi_{j}^{\prime}(s) \mathrm{d} s \\
& =\sum_{j=1}^{m}\left(\left.b_{i_{1} j}(s) \varphi_{j}(s)\right|_{1} ^{t_{1}}-\int_{1}^{t_{1}} \sum_{j, k=1}^{m} b_{i_{1} j k}(s) u_{k}(s) \varphi_{j}(s) \mathrm{d} s\right) .
\end{aligned}
$$

Here, the $b_{i_{1} j k}$ denote some components of $D \mathcal{A}^{-1}$. Therefore, by (66) and (68), we have

$$
\frac{1}{6} \leq 2 m D \rho+D \sum_{j, k=1}^{m} \int_{t_{1}}^{1}\left|u_{k}(s) \varphi_{j}(s)\right| \mathrm{d} s
$$

and, with $\rho \leq \frac{1}{24 m D}$, we have $\int_{t_{1}}^{1}\left|u_{k}(s) \varphi_{j}(s)\right| \mathrm{d} s \geq \frac{1}{12 m^{2} D}$, for some $j, k \in(1, \cdots, m)$. Hence, (65) follows.

1.c.: Equality in $\left(P_{3}\right)$ : There exist $t_{*}<t^{*} \in\left[t_{1}, 1\right]$, such that

$$
\left\|\varphi\left(t^{*}\right)-\varphi\left(t_{*}\right)\right\| \geq \frac{\rho}{4} \text { and } \forall t \in\left[t_{*}, t^{*}\right],\|\varphi(t)\| \geq \frac{\rho}{4}
$$


Therefore, using Cauchy-Schwarz inequality, we obtain

$$
\begin{aligned}
\|\varphi\|_{L^{2}\left(t_{*}, t^{*}\right)}\|u\|_{L^{2}\left(t_{*}, t^{*}\right)} & \geq \frac{\rho}{4}\left(t_{*}-t^{*}\right)^{1 / 2}\|u\|_{L^{2}\left(t_{*}, t^{*}\right)} \\
& \geq \frac{\rho}{4 D}\left\|\int_{t_{*}}^{t^{*}} \mathcal{A}(t) u(t) \mathrm{d} t\right\|=\frac{\rho}{4 D}\left\|\varphi\left(t^{*}\right)-\varphi\left(t_{*}\right)\right\| \geq \frac{\rho^{2}}{16 D},
\end{aligned}
$$

and (65) follows.

- Case 2. $\left(P_{4}\right)$ holds.

2.a. Equality in $\left(P_{1}\right)$ : the conclusion of the theorem follows from Theorem 4 of [31].

2.b. Equality in $\left(P_{2}\right)$ : by $\left(P_{4}\right)$, there exist a time $t_{1}^{\prime}>t_{1}$ and an index $i_{1}$ in $1, \cdots, m$, such that

$$
\frac{1}{12} \leq\left|U_{i_{1}}(t)\right| \leq \frac{1}{6} \text { for } t \in\left[t_{1}, t_{1}^{\prime}\right] \text { and }\left|U_{i_{1}}\left(t_{1}^{\prime}\right)\right|=\frac{1}{12}
$$

Therefore, using $\left(P_{4}\right)$ and Cauchy-Schwarz inequality in equation (70), we have

$$
\begin{gathered}
\|\varphi\|_{L^{2}\left(t_{1}, t_{1}^{\prime}\right)}\|u\|_{L^{2}\left(t_{1}, t_{1}^{\prime}\right)} \geq \frac{\rho}{4}\left(t_{1}^{\prime}-t_{1}\right)^{1 / 2}\|u\|_{L^{2}\left(t_{1}, t_{1}^{\prime}\right)} \\
\geq \frac{\rho}{4}\left|\int_{t_{1}}^{t_{1}^{\prime}} u_{i_{1}}(s) d s\right| \geq \frac{\rho}{4}\left|U_{i_{1}}\left(t_{1}\right)-U_{i_{1}}\left(t_{1}^{\prime}\right)\right| \geq \frac{\rho}{48}
\end{gathered}
$$

which implies (65).

2.c. Equality in $\left(P_{4}\right)$ : this case reduces to 1.c. Indeed, equation (69) still holds.

Taking into account Lemma 6 , the proof of Theorem 4 reduces to show the next lemma.

Lemma 7. There exists $C>0$ such that for every $u \in \mathcal{H}$ and $z \in T_{\mathcal{E}(u)}^{*} M$ so that $(\mathcal{E}(u), z) \in \mathcal{K}$, if $t_{1} \in[0,1)$ is the "first exit time" corresponding to $(u, z)$, then we have

$$
\left\|u^{(r)}\right\|_{L^{2}\left(\left[t_{1}, 1\right]\right)}\left\|R^{(r)}\right\|_{L^{2}\left(\left[t_{1}, 1\right]\right)} \geq C\|u\|_{L^{2}\left(\left[t_{1}, 1\right]\right)}\|\varphi\|_{L^{2}\left(\left[t_{1}, 1\right]\right)}
$$

Proof of Lemma \%. We need two lemmas given next.

Lemma 8 (cf. Adams [1]). Let $f=\left(f_{1}, \cdots, f_{p}\right):[a, b] \rightarrow \mathbb{R}^{p}$, where $p$ is a positive integer, and each $f_{i}$ has an absolutely continuous first derivative; let $l \stackrel{\text { def }}{=} b-a>0$ and

$$
M_{j} \stackrel{\text { def }}{=}\left(\sum_{i=1}^{p}\left\|f_{i}^{(j)}\right\|_{L^{2}([a, b])}^{2}\right)^{1 / 2}
$$

for $j=0,1,2$. Then there exists a universal constant $C>0$ such that

(L2i) if $M_{0} \leq l^{2} M_{2}$, then $M_{1}^{2} \leq M_{0} M_{2}$;

(L2ii) if $M_{0}>l^{2} M_{2}$, then $M_{1} \leq C \frac{M_{0}}{l}$.

Lemma 9. Let $f:[a, b] \rightarrow \mathbb{R}$ be an absolutely continuous function such that $f(b)=0$ and $\sup _{t \in[a, b]}\left|\int_{b}^{t} f(s) d s\right| \leq$ $\frac{1}{6}$. Then

$$
\int_{a}^{b} f^{4}(s) d s \leq \int_{a}^{b} f^{\prime}(s)^{2} \mathrm{~d} s .
$$


Proof of Lemma 9. Define $I(t)=\int_{a}^{t} f(s) \mathrm{d} s$. Then,

$$
\forall t \in[a, b],|I(t)| \leq 1 / 3 .
$$

Therefore, integrating by parts and using (72), we get

$$
\int_{a}^{b} f^{4}(s) \mathrm{d} s=\left.I(s) f^{3}(s)\right|_{s=a} ^{s=b}-3 \int_{a}^{b} I(s) f^{2}(s) f^{\prime}(s) \mathrm{d} s \leq \int_{a}^{b} f^{2}(s)\left|f^{\prime}(s)\right| \mathrm{d} s .
$$

Then, we conclude using Cauchy-Schwarz inequality.

We will also need some obvious facts: if $q$ is a positive integer, then

$$
\begin{gathered}
\forall v \in H^{q, 1}, \forall t \in[0,1], v(t)=\int_{1}^{t} \frac{(t-s)^{q-1}}{(q-1) !} v^{(q)}(s) \mathrm{d} s, \\
\|v\|_{L^{2}(t, 1)} \leq C_{q}(1-t)^{q}\left\|v^{(q)}\right\|_{L^{2}([t, 1])}
\end{gathered}
$$

with $C_{q}=\frac{1}{\sqrt{2 q(2 q-1)}(q-1) !} \leq \frac{1}{\sqrt{2}}$. In particular, for $u \in \mathcal{H}$ and $l \stackrel{\text { def }}{=} 1-t_{1}$, we have

$$
\|u\|_{L^{2}\left(\left[t_{1}, 1\right]\right)} \leq C_{r} l^{r}\left\|u^{(r)}\right\|_{L^{2}\left(\left[t_{1}, 1\right]\right)} .
$$

Therefore, for $r \geq 2$, every $u \in \mathcal{H}$ satisfies $(L 2 i)$.

From now on, we fix $u \in \mathcal{H}$ and $z \in T_{\mathcal{E}(u)}^{*} M$ so that $(\mathcal{E}(u), z) \in \mathcal{K}$. Let $l \stackrel{\text { def }}{=} 1-t_{1}(>0)$, where $t_{1}$ is defined in Lemma 5. Recall that (65) holds. In order to prove (71), several cases are to be considered:

Case 1. $\left(P_{3}\right)$ holds.

1.a. (L2ii) holds for $R^{(2 r)}$.

1.b. ( $L 2 i i)$ holds for $R^{\left(r+j_{0}\right)}$, with $j_{0}<r$.

1.c. ( $L 2 i i)$ does not hold for any $R^{(r+j)}$, where $j \in\{0, \cdots, r\}$.

Case 2. $\left(P_{4}\right)$ holds.

We show in Case $1 a$, that actually ( $L 2 i i)$ holds for every $R^{(r+j)}$, where $j \in\{0, \cdots, r\}$. In Case $1 b$, we choose the largest $j_{0}<r$ for which $(L 2 i i)$ holds and then show that $(L 2 i i)$ holds for every $j \leq j_{0}$.

Proof of Case 1. It is clear that $\mathcal{A}(t)$ is invertible on $\left[t_{1}, 1\right]$. Using (54), we have

$$
\forall t \in\left[t_{1}, 1\right], u(t)=\mathcal{A}^{-1}(t) \varphi^{\prime}(t) .
$$

From (54) and (76), we get

$$
\frac{1}{C_{\mathcal{K}}}\|u\|_{L^{2}\left(\left[t_{1}, 1\right]\right)} \leq\left\|\varphi^{\prime}\right\|_{L^{2}\left(\left[t_{1}, 1\right]\right)} \leq C_{\mathcal{K}}\|u\|_{L^{2}\left(\left[t_{1}, 1\right]\right)} .
$$

In addition, for $i=1, \cdots, m$, and $t \in\left[t_{1}, 1\right]$, we have

$$
\varphi^{\prime \prime}(t)=\mathcal{A}(t) u^{\prime}(t)+\mathcal{A}^{\prime}(t) u(t) .
$$

Since $\varphi_{i, j}^{\prime}(t)=\sum_{k=1}^{m} \varphi_{i, j, k}(t) u_{k}(t)$, we have

$$
\left\|\varphi^{\prime \prime}(t)\right\| \leq C_{\mathcal{K}}\left(\left\|u^{\prime}(t)\right\|+\left|u^{T} u(t)\right|\right),
$$


where $C_{\mathcal{K}}$ is a constant depending on $\mathcal{K}$. By Lemma 9 , we obtain

$$
\left\|\varphi^{\prime \prime}\right\|_{L^{2}\left(\left[t_{1}, 1\right]\right)} \leq C_{\mathcal{K}}\left\|u^{\prime}\right\|_{L^{2}\left(\left[t_{1}, 1\right]\right)}
$$

We now apply lemma 8 successively to $f:=R^{(r)}, \cdots, f:=R^{(2 r)}=(-1)^{r} \varphi$.

Case 1.a. We have

$$
\left\|R^{(2 r+1)}\right\|_{L^{2}\left(\left[t_{1}, 1\right]\right)}=\left\|\varphi^{\prime}\right\|_{L^{2}\left(\left[t_{1}, 1\right]\right)} \leq C_{\mathcal{K}} \frac{\left\|R^{(2 r)}\right\|_{L^{2}\left(\left[t_{1}, 1\right]\right)}}{l}=C_{\mathcal{K}} \frac{\|\varphi\|_{L^{2}\left(\left[t_{1}, 1\right]\right)}}{l}
$$

Using (77), we obtain

$$
\|u\|_{L^{2}\left(\left[t_{1}, 1\right]\right)} \leq C_{\mathcal{K}} \frac{\|\varphi\|_{L^{2}\left(\left[t_{1}, 1\right]\right)}}{l} .
$$

We need the following lemma, whose proof is postponed to the end of the section.

Lemma 10. Assume that there exists $j_{0} \leq r$, such that

$$
\left\|R^{\left(r+j_{0}+1\right)}\right\|_{L^{2}\left(\left[t_{1}, 1\right]\right)} \leq C_{\mathcal{K}} \frac{\left\|R^{\left(r+j_{0}\right)}\right\|_{L^{2}\left(\left[t_{1}, 1\right]\right)}}{l}
$$

Then, for $0 \leq j \leq j_{0}$, we have

$$
\left\|R^{(r+j+1)}\right\|_{L^{2}\left(\left[t_{1}, 1\right]\right)} \leq C_{\mathcal{K}} \frac{\left\|R^{(r+j)}\right\|_{L^{2}\left(\left[t_{1}, 1\right]\right)}}{l}
$$

From Lemma 10 applied to $j_{0}=r$, we obtain

$$
\|\varphi\|_{L^{2}\left(\left[t_{1}, 1\right]\right)} \leq C_{\mathcal{K}} \frac{\left\|R^{(r)}\right\|_{L^{2}\left(\left[t_{1}, 1\right]\right)}}{l^{r}}
$$

Multiplying (82) and (75), we obtain the estimate (71).

Case 1.b. From Lemma 10, we have

$$
\left\|R^{\left(r+j_{0}+1\right)}\right\|_{L^{2}\left(\left[t_{1}, 1\right]\right)} \leq C_{\mathcal{K}} \frac{\left\|R^{(r)}\right\|_{L^{2}\left(\left[t_{1}, 1\right]\right)}}{l^{j_{0}+1}}
$$

If $j_{0}=r-1$, then (82) and (75) hold. Hence, we get the estimate (71). If $j_{0}<r-1$, we apply lemma 8 successively to $f:=R^{\left(r+j_{0}+l\right)}$, for $l=1, \cdots, r-j_{0}$. For $j \leq j_{0}$, we are in the case $(L 2 i)$ and then, we get for $l=1, \cdots, r-j_{0}$ that

$$
\left\|R^{\left(r+j_{0}+l+1\right)}\right\|_{L^{2}\left(\left[t_{1}, 1\right]\right)}^{2} \leq C\left\|R^{\left(r+j_{0}+l\right)}\right\|_{L^{2}\left(\left[t_{1}, 1\right]\right)}\left\|R^{\left(r+j_{0}+l+2\right)}\right\|_{L^{2}\left(\left[t_{1}, 1\right]\right)}
$$

Thanks to (77) and (78), we can write (84) for $l=r-j_{0}$ as

$$
\|u\|_{L^{2}\left(\left[t_{1}, 1\right]\right)}^{2} \leq C_{\mathcal{K}}\|\varphi\|_{L^{2}\left(\left[t_{1}, 1\right]\right)}\left\|u^{\prime}\right\|_{L^{2}\left(\left[t_{1}, 1\right]\right)} .
$$

We elevate (84) to the power $l$, for $l=1, \cdots, r-j_{0}-1$, and (85) to the power $r-j_{0}-1$. We multiply together all the inequalities previously obtained and get

$$
\|u\|_{L^{2}\left(\left[t_{1}, 1\right]\right)}^{r-j_{0}-1}\|\varphi\|_{L^{2}\left(\left[t_{1}, 1\right]\right)} \leq C_{\mathcal{K}}\left\|R^{\left(r+j_{0}+l\right)}\right\|_{L^{2}\left(\left[t_{1}, 1\right]\right)}\left\|u^{\prime}\right\|_{L^{2}\left(\left[t_{1}, 1\right]\right)}^{\left(r-j_{0}-1\right)}
$$


On the other hand, we apply Lemma 8 to successively $f:=u^{l}$ for $l=0, \cdots, r-j_{0}-3$. Since $C_{q}<1$ for $q \geq 1$, we are in the case $(L 2 i)$. Therefore, we have, for $l=0, \cdots, r-j_{0}-3$, after elevating to the power $r-j_{0}-2-l$,

$$
\left\|u^{l+1}\right\|_{L^{2}\left(\left[t_{1}, 1\right]\right)}^{2\left(r-j_{0}-2-l\right)} \leq C\left\|u^{l}\right\|_{L^{2}\left(\left[t_{1}, 1\right]\right)}^{r-j_{0}-2-l}\left\|u^{l+2}\right\|_{L^{2}\left(\left[t_{1}, 1\right]\right)}^{r-j_{0}-2-l} .
$$

Multiplying the previous inequalities, we obtain

$$
\left\|u^{\prime}\right\|_{L^{2}\left(\left[t_{1}, 1\right]\right)}^{\left(r-j_{0}-1\right)} \leq C\|u\|_{L^{2}\left(\left[t_{1}, 1\right]\right)}^{r-j_{0}-2}\left\|u^{\left(r-j_{0}-1\right)}\right\|_{L^{2}\left(\left[t_{1}, 1\right]\right)} .
$$

Multiplying (86) and (88), we end up with

$$
\|\varphi\|_{L^{2}\left(\left[t_{1}, 1\right]\right)}\|u\|_{L^{2}\left(\left[t_{1}, 1\right]\right)} \leq C_{\mathcal{K}}\left\|R^{\left(r+j_{0}+1\right)}\right\|_{L^{2}\left(\left[t_{1}, 1\right]\right)}\left\|u^{\left(r-j_{0}-1\right)}\right\|_{L^{2}\left(\left[t_{1}, 1\right]\right)} .
$$

Using (74) (for $v:=u^{\left(r-j_{0}-1\right)}$ and $q:=j_{0}+1$ ), (83) and (89), we obtain the estimate (71).

Case 1.c. For every $j=0, \cdots, r,(L 2 i)$ holds. This case reduces to case 1.b. with $j_{0}=-1$.

Proof of Case 2. Using the following inequality:

$$
\|U\|_{L^{2}\left(\left[t_{1}, 1\right]\right)} \leq \mu \sqrt{m l}=\frac{4 \mu}{\rho} \frac{\rho}{4} \sqrt{m l} \leq \frac{4 \mu \sqrt{m}}{\rho}\|\varphi\|_{L^{2}\left(\left[t_{1}, 1\right]\right)}
$$

and applying lemma 8 to $U$, we obtain (note that $U \in H^{r+1, m}$ )

$$
\|u\|_{L^{2}\left(\left[t_{1}, 1\right]\right)}^{2} \leq C\|U\|_{L^{2}\left(\left[t_{1}, 1\right]\right)}\left\|u^{\prime}\right\|_{L^{2}\left(\left[t_{1}, 1\right]\right)}
$$

Then, thanks to (90), we obtain $\|u\|_{L^{2}\left(\left[t_{1}, 1\right]\right)}^{2} \leq C_{\mathcal{K}}\|\varphi\|_{L^{2}\left(\left[t_{1}, 1\right]\right)}\left\|u^{\prime}\right\|_{L^{2}\left(\left[t_{1}, 1\right]\right)}$ and we are back to case 1.b. The proof of Lemma 7 is complete.

Proof of Lemma 10. The proof goes by "backward" induction on $j$. For $j=j_{0}$, (81) holds. Suppose the conclusion is true for some $1 \leq j \leq j_{0}$. Apply Lemma 8 to $f:=R^{(r+j-1)}$. If $(L 2 i i)$ holds we are done, otherwise

$$
\left\|R^{(r+j)}\right\|_{L^{2}\left(\left[t_{1}, 1\right]\right)}^{2} \leq C\left\|R^{(r+j-1)}\right\|_{L^{2}\left(\left[t_{1}, 1\right]\right)}\left\|R^{(r+j+1)}\right\|_{L^{2}\left(\left[t_{1}, 1\right]\right)} .
$$

By the induction hypothesis, we obtain

$$
\left\|R^{(r+j)}\right\|_{L^{2}\left(\left[t_{1}, 1\right]\right)}^{2} \leq C\left\|R^{(r+j-1)}\right\|_{L^{2}\left(\left[t_{1}, 1\right]\right)} C_{\mathcal{K}} \frac{\left\|R^{(r+j)}\right\|_{L^{2}\left(\left[t_{1}, 1\right]\right)}}{l},
$$

which implies

Lemma 10 is proved.

$$
\left\|R^{(r+j)}\right\|_{L^{2}\left(\left[t_{1}, 1\right]\right)} \leq C_{\mathcal{K}} \frac{\left\|R^{(r+j-1)}\right\|_{L^{2}\left(\left[t_{1}, 1\right]\right)}}{l}
$$

\section{Conclusion}

In this paper, we have presented in details how the well-known continuation method applies to the motionplanning problem for a nonlinear control-affine system without drift on a smooth, finite-dimensional Riemannian manifold. In the context of nonlinear geometric control, that idea goes back to Sussmann (cf. [32]). The continuation method comes down to the study of the so-called "PLE", which is an ordinary differential equation in the space of controls. More precisely, there are two technical obstacles associated with the continuation method: $(i)$ avoidance of the singular set on which the differential of the end-point mapping looses surjectivity 
(and on which the PLE is not defined); (ii) possible explosions in finite time of solutions of the PLE. The results obtained in the present paper can be divided in two sets. In the first one, general facts regarding the PLE are established: $(1-a)$ if the PLE admits a global solution in a control space, then there exists a finite-dimensional space where the corresponding PLE also admits a global solution; $(1-b)$ the maximal solution of the PLE is as regular as its initial condition. The second set of results concern control-affine system without drift verifying the Strong Bracket Generating Condition. For such control systems, Sussmann proved an existence theorem for the global solution of the PLE in the space of controls with finite $L^{2}$-norm ( $c f$.[31]). We have extended that result to other spaces of controls, which are more regular.

\section{Appendix}

\subsection{Property $(C l)_{k}$}

A map $u:[0,1] \rightarrow \mathbb{R}$ is piecewise-constant if there exist an integer $p \geq 1$ and $t_{1}<\cdots<t_{p}$ in $[0,1]$ such that $u$ is constant on the intervals $\left[0, t_{1}\right), \cdots,\left(t_{p-1}, t_{p}\right),\left(t_{p}, 1\right]$. We use $\mathcal{U}_{\text {step }}$ to denote the set of all piecewise-constant maps on $[0,1]$. For every set $F \in \mathbb{R}$, we use $\mathcal{U}_{\text {step }}^{F}$ to denote the set of the piecewise-constant maps on $[0,1]$ taking values in $F$. We use $\Delta_{p}$ to denote the set of $p$-tuples $\left(t_{1}, \cdots, t_{p}\right)$ such that $t_{i} \geq 0$ for $i=1, \cdots, p$ and $t_{1}+\cdots+t_{p} \leq 1$. For the real numbers $\omega_{1}, \cdots, \omega_{p+1}$ and $\left(t_{1}, \cdots, t_{p}\right) \in \Delta_{p}$, we associate the piecewise-constant map on $[0,1]$

$$
\left[\omega_{1}, t_{1}\right] * \cdots *\left[\omega_{p}, t_{p}\right] * \omega_{p+1},
$$

equal to $\omega_{1}$ on $\left[0, t_{1}\right), \omega_{1}$ on $\left[t_{1}, t_{1}+t_{2}\right), \cdots, \omega_{p}$ on $\left[t_{1}+\cdots+t_{p-1}, t_{1}+\cdots+t_{p}\right)$ and $\omega_{p+1}$ on $\left[t_{1}+\cdots+t_{p}, 1\right)$.

Definition 2 ( $c f$. Def. 4.5 of Grasse and Sussmann [12]). Let $\mathcal{W}$ be a specified nonempty subset of $\mathcal{U}_{\text {step. An }}$ admissible sequence of approximation operators $\left(P_{j}\right)$ for $\mathcal{W}$ is a sequence of maps $P_{j}: \mathcal{U}_{\text {step }} \rightarrow \mathcal{W}(j \in \mathbb{N})$ such that:

(i) for every finite subset $F$ of real numbers there exists a compact set $\Omega$ of real numbers such that $F \subset \Omega$ and, for every $j \in \mathbb{N}$,

$$
P_{j}\left(\mathcal{U}_{\text {step }}^{F}\right) \subset \mathcal{W} \cap \mathcal{U}_{\text {step }}^{\Omega}
$$

(ii) for every $j, p \in \mathbb{N}$ and for every real numbers $\omega_{1}, \cdots, \omega_{p+1}$, the map

$$
\left(t_{1}, \cdots, t_{p}\right) \rightarrow P_{j}\left(\left[\omega_{1}, t_{1}\right] * \cdots *\left[\omega_{p}, t_{p}\right] * \omega_{p+1}\right)
$$

from $\Delta_{p}$ to $\mathcal{W}$ is continuous in the topology of convergence in measure on $[0,1]$;

(iii) for $p \in \mathbb{N}$, for every real numbers $\omega_{1}, \cdots, \omega_{p+1}$ and for every $\left(t_{1}, \cdots, t_{p}\right) \in \Delta_{p}$, we have

$$
\lim _{j \rightarrow \infty} P_{j}\left(\left[\omega_{1}, t_{1}\right] * \cdots *\left[\omega_{p}, t_{p}\right] * \omega_{p+1}\right)=\left[\omega_{1}, t_{1}\right] * \cdots *\left[\omega_{p}, t_{p}\right] * \omega_{p+1},
$$

and the convergence is locally uniform in $\left(t_{1}, \cdots, t_{p}\right)$.

Definition 3. For $k \geq 0$, a closed linear subspace $\mathcal{H}$ of $H^{k}([0,1], \mathbb{R})$ has the $(C l)_{k}$ property if either $k=0$ and $\mathcal{H}=L^{2}([0,1])$ or $k \geq 1$ and there exists an admissible sequence of approximation operators $\left(P_{j}\right)$ for $\mathcal{H}$.

We have

Proposition 5. For $r \geq 1, H^{r, 1}$ verifies Property $(\mathrm{Cl})_{r}$.

Proof of Proposition 5. For $r=0, \mathcal{U}_{\text {step }} \subset L^{2}([0,1])$. Then it is enough to take the $P_{j}$ 's to be the inclusion operators. We now assume that $r \geq 1$. For $\rho>0$, let $\lambda_{\rho}: \mathbb{R} \rightarrow \mathbb{R}$ be a mollifier i.e. $\lambda_{\rho}$ is a smooth function such that $\lambda_{\rho}(t)>0$ for $t \in[-\rho, \rho], \lambda_{\rho}(t)=0$ for $t \in \mathbb{R} \backslash[-\rho, \rho]$ and $\int_{-\infty}^{\infty} \lambda_{\rho}(t) \mathrm{d} t=1$. For $\rho>0$ and $u \in \mathcal{U}_{\text {step }}$, let $u_{\rho}$ be the function defined on $[0,1]$ by

$$
u_{\rho}(t)=\frac{(1-t)^{r}}{(1-t)^{r}+\rho} \int_{-\rho}^{\rho} u(t-s) \lambda_{\rho}(s) \mathrm{d} s
$$


where $u$ is taken to be 0 outside $[0,1]$. Since $u$ is bounded, $u_{\rho}$ tends to $u$ in $L^{2}$-norm as a consequence of Fubini theorem and the dominated convergence theorem. Therefore, by choosing a decreasing sequence $\left(\rho_{j}\right)_{j \in \mathbf{N}}$ of positive numbers that tends to 0 , we get an admissible sequence of approximation operators $\left(P_{j}\right)$ for $H^{r, 1}$, where each $\left(P_{j}\right)$ associates to an element $u \in \mathcal{U}_{\text {step }}$ the input $u_{\rho_{j}} \in H^{r, 1}$.

\subsection{Proof of equation (20)}

We want to establish that there exists an open bounded subset $U_{K}$ of $M$ containing $K$ such that, for every smooth vector field $B \in V^{\infty}(M)$, there exist $r$ smooth real-valued functions $v_{1, B}, \cdots, v_{r, B}$ defined on $U_{K}$ for which (20) holds for every $x \in U_{K}$.

We fix an open bounded subset $U_{K}$ of $M$ containing $K$ such that, for every $x \in \overline{U_{K}},(19)$ holds. Then, since $\overline{U_{K}}$ is compact, we can find a finite number of bounded open subsets $\left(U_{j}\right)_{j \in J}$ of $M$ and charts $\left(\left(x_{j}\right), \operatorname{Dom}\left(x_{j}\right)\right)_{j \in J}$ such that for $j \in J, \overline{U_{j}} \subset \operatorname{Dom}\left(x_{j}\right)$, and for every $x \in \overline{U_{j}}$, (19) holds. For $j \in J$ and $x \in \overline{U_{j}}$, we can consider $F^{(j)}(x)$, the $n \times r$ matrix whose columns are the $f_{\ell}(x)^{\prime}$ 's, $\ell=1, \cdots, r$, written using the chart $\left(\left(x_{j}\right), \operatorname{Dom}\left(x_{j}\right)\right)$. Since rank $F^{(j)}(x)=n$, we can define a pseudo-inverse $P_{F^{(j)}}(x)$ of $F^{(j)}(x)$ as

$$
P_{F^{(j)}}(x)=F^{(j)}(x)^{T}\left(F^{(j)}(x) F^{(j)}(x)^{T}\right)^{-1}
$$

In particular, we have $F^{(j)}(x) P_{F^{(j)}}(x)=I_{T_{x} M}$. Then, for $B \in V^{\infty}(M)$ and $x \in \overline{U_{j}}$, we express $B(x)$ using the chart $x_{j}$ and obtain

$$
B(x)=F^{(j)}(x) P_{F^{(j)}}(x) B(x)=\sum_{\ell=1}^{r} v_{\ell, B}^{(j)}(x) f_{\ell}(x),
$$

where the $r$ real-valued functions $v_{1, B}^{(j)}, \cdots, v_{r, B}^{(j)}$ are smooth on $U_{j}$. We will denote the previous expression $B^{(j)}(x)$. We now multiply $B$ by a smooth function $\psi^{(j)}$ compactly supported in $\operatorname{Dom}\left(x_{j}\right)$, such that $0 \leq \psi^{(j)} \leq 1$ and $\psi^{(j)} \equiv 1$ near $\overline{U_{j}}$. Then, for $x \in U_{K}$, the vector field $\tilde{B}$ is defined by

$$
\tilde{B}(x)=\sum_{j \in J} f^{(j)}(x) \psi^{(j)}(x) B^{(j)}(x),
$$

where $\left(f^{(j)}\right)_{j \in J}$ is a partition of unity subordinate to the open covering of $\overline{U_{K}},\left(U_{j}\right)_{j \in J}$. It is clear that $\tilde{B}$ and $B$ coincide on $U_{K}$ and, by rearranging (93), we have that for $x \in U_{K}, B(x)=\sum_{\ell=1}^{r} v_{\ell, B}(x) f_{\ell}(x)$, where $v_{\ell, B}(x)=\sum_{j \in J} f^{(j)}(x) \psi^{(j)}(x) v_{\ell, B}^{(j)}(x), \ell=1, \cdots, r$.

\subsection{Existence of $h$ in the proof of Proposition 3}

Let $\pi:[0,1] \rightarrow M$ be a $C^{1}$ curve. Consider $K=\pi([0,1])$ and $V$ a bounded open set of $M$ containing $K$. We show the existence of a time-varying vector field $\{h(s, \cdot)\}_{s \in[0,1]}$ such that

(i) $h$ is continuous on $[0,1] \times M$ and $h(s, \cdot)$ is smooth for every $s \in[0,1]$;

(ii) for every $x \in M / V, h(\cdot, x):[0,1] \rightarrow T_{x} M$ is identically equal to 0 ;

(iii) $\forall s$ in $[0,1], h(s, \pi(s))=\frac{\mathrm{d} \pi}{\mathrm{d} s}(s)$ and $\exists C>0, \forall x$ in $M,\|h(s, x)\| \leq\left\|\frac{\mathrm{d} \pi}{\mathrm{d} s}(s)\right\|$ and $\left\|D_{x} h(s, x)\right\| \leq C$.

Since $[0,1]$ is compact, we can find a finite number of relatively open subintervals of $[0,1],\left(I_{j}\right)_{j \in J}$ and charts $\left(\left(x_{j}\right), \operatorname{Dom}\left(x_{j}\right)\right)_{j \in J}$ such that for $j \in J, \overline{\pi\left(I_{j}\right)} \subset \operatorname{Dom}\left(x_{j}\right)$. Then, for $j \in J s \in I_{j}$ and $x \in \operatorname{Dom}\left(x_{j}\right)$, define $h_{j}(s, x)$ as the constant vector field with value $\frac{\mathrm{d} \pi}{\mathrm{d} s}$. We now multiply $h_{j}$ by a smooth function $\psi_{j}$ compactly supported in $\operatorname{Dom}\left(x_{j}\right)$ such that $0 \leq \psi_{j} \leq 1$ and $\psi_{j} \equiv 1$ near $\pi\left(I_{j}\right)$. Then, $h(s, x)$ is taken as $\sum_{j \in J} f_{j}(x) \psi_{j}(x) h_{j}(s, x)$, where $\left(f_{j}\right)_{j \in J}$ is a partition of unity subordinate to the open covering of $[0,1],\left(I_{j}\right)_{j \in J}$. Furthermore, there exists a bounded open subset of $M$ such that, for every $s \in[0,1], h(s, \cdot)$ is identically equal to 0 . It is then clear that conditions $(i)$ to $($ iii $)$ hold. 


\subsection{Existence of the function $\psi$ used for the MPPO}

Let $C$ be a closed subset of the complete Riemannian manifold $(M,\langle\cdot, \cdot\rangle)$. Let us first assume that there exists a smooth function $\psi: M \rightarrow \mathbb{R}$ such that $\psi \equiv 0$ on $C, 0<\psi(x) \leq \inf (1, d(x, C))$ on $M \backslash C$ and $\sup _{x \in M} \sum_{j=1}^{3}\left\|D^{j} \psi(x)\right\|$ is finite. We use $\bar{d}$ to denote the distance function on $M \backslash C$ induced by the metric $\frac{\langle\cdot, \cdot\rangle}{\psi^{2}}$. Suppose that it is not the case. Therefore, there exists a geodesic $x:\left[0, t_{0}\right) \rightarrow M \backslash C$ such that $t_{0}$ is finite and $x(t)$ tends to some point $p \in C$ as $t$ goes to $t_{0}$. Let us assume that, in addition, the arc $x$ is parameterized by arclength. If $J(t) \stackrel{\text { def }}{=} \int_{t}^{t_{0}} \sqrt{\langle\dot{x}(s), \dot{x}(s)\rangle} \mathrm{d} s$ for $t \in\left[0, t_{0}\right)$, then $\dot{J}(t)=-\langle\dot{x}(t), \dot{x}(t)\rangle$ and $\psi(x(t)) \leq d(p, x(t)) \leq J(t)$. For $t \in\left[0, t_{0}\right)$, we have

$$
t_{0} \geq t=\int_{0}^{t} \frac{\sqrt{\langle\dot{x}(s), \dot{x}(s)\rangle}}{\psi(x(s))} \mathrm{d} s \geq \int_{0}^{t} \frac{-\dot{J}(s)}{J(s)} \mathrm{d} s=\ln \frac{J(0)}{J(t)} .
$$

Since $\lim _{t \rightarrow t_{0}^{-}} J(t)=0$, we get $t_{0}=+\infty$, which is a contradiction. Then $\left(M \backslash C, \frac{\langle\cdot, \cdot\rangle}{\psi^{2}}\right)$ is complete.

To construct the required function $\psi$, consider an increasing sequence $\left(K_{i}\right)_{i \geq 0}$ of compact subsets of $M$ such that $C=\cup_{i \geq 0} K_{i}$. For every $i \geq 0$, we can construct a smooth function $\psi_{i}: M \rightarrow \mathbb{R}$ such that $\psi_{i} \equiv 0$ on $K_{i}$ and $0<\psi_{i}(x) \leq \inf \left(1, d\left(x, K_{i}\right)\right)$ for $x \in M \backslash K_{i}$. Let $M_{i} \stackrel{\text { def }}{=} \sup _{x \in M} 1+\sum_{j=1}^{j=3}\left\|D^{j} \psi_{i}(x)\right\|$. We then define $\psi$ as $\sum_{i \geq 0} \frac{1}{2^{i} M_{i}} \psi_{i}$.

\section{REFERENCES}

[1] R.A. Adams, Sobolev Spaces. Academic Press, New York (1975).

[2] E.L. Allgower and K. Georg, Continuation and Path Following. Acta Numerica (1992).

[3] J.M. Bismuth, Large Deviations and the Malliavin Calculus. Birkhäuser (1984).

[4] L. Cesari, Functional analysis and Galerkin's method. Mich. Math. J. 11 (1964) 385-418.

[5] A. Chelouah and Y. Chitour, On the controllability and trajectories generation of rolling surfaces. Forum Math. 15 (2003) $727-758$.

[6] Y. Chitour, Applied and theoretical aspects of the controllability of nonholonomic systems. Ph.D. thesis, Rutgers University (1996).

[7] Y. Chitour, Path planning on compact Lie groups using a continuation method. Syst. Control Lett. 47 (2002) $383-391$.

[8] Y. Chitour and H.J. Sussmann, Line-integral estimates and motion planning using a continuation method. Essays on Math. Robotics, J. Baillieul, S.S. Sastry and H.J. Sussmann Eds., IMA. Math. Appl. 104 (1998) 91-125.

[9] S.N. Chow and J.K. Hale, Methods of Bifurcation Theory. Springer, New York 251 (1982).

[10] A. Divelbiss and J.T. Wen, A Path Space Approach to Nonholonomic Motion Planning in the Presence of Obstacles. IEEE Trans. Robotics Automation 13 (1997) 443-451.

[11] Ge Zhong, Horizontal Path Spaces and Carnot-Carathéodory Metrics. Pacific J. Math. 161 (1993) 255-286.

[12] K.A. Grasse and H.J. Sussmann, Global controllability by nice controls, Nonlinear controllability and optimal control. Dekker, NY. Mono. Text. Pure Appl. Math. 133 (1990) 33-79.

[13] J.K. Hale, Applications of alternative problems. Lectures notes, Brown University (1971).

[14] M.W. Hirsch, Differential Topology. Springer, New York (1976).

[15] V. Jurdjevic, Geometric control theory. Cambridge Studies in Adv. Math., Cam. Univ. Press (1997).

[16] G. Lafferriere, and H.J. Sussmann, Motion planning for controllable systems without drift, in Proc. Int. Conf. Robot. Auto. Sacramento, CA (1991) 1148-1153.

[17] E.B. Lee and L. Markus, Foundations of Optimal Control Theory. Wiley, New York (1967).

[18] J. Leray and J. Schauder, Topologie et équations fonctionelles. Ann. Sci. Ecole Norm. Sup. 51 (1934) 45-78.

[19] T.Y. Li, Numerical solution of multivariate polynomial systems by homotopy continuation methods. Acta Numerica (1997) 399-436.

[20] W. Liu, An approximation algorithm for nonholonomic systems. SIAM J. Control Optim. 35 (1997) 1328-1365.

[21] W. Liu and H.J. Sussmann, Shortest paths for sub-Riemannian metrics on rank 2 distributions. Memoirs of the AMS, \#564 118 (1995).

[22] P. Martin, Contribution à l'étude des systèmes différentiellement plats. Ph.D. thesis, École des Mines de Paris, Paris, France (1992).

[23] R. Montgomery, Abnormal Optimal Controls and Open Problems in Nonholonomic Steering. J. Dyn. Cont. Sys. 1 Plenum Pub. Corp. (1995) 49-90.

[24] R.M. Murray and S.S. Sastry, Steering nonholonomic systems using sinusoids, in Proc. IEEE Conference on Decision and Control (1990). 
[25] Cz. Olech, On the Wazewski equation, in Proc. of the conference, Topological methods in Differential Equations and Dynamical systems, Krakow (1996). Univ. Iagel. Acta Math. 36 (1998) 55-64.

[26] P. Pansu, Métriques de Carnot-Carathéodory et quasiisométries des espaces symétriques de rang un. Ann. Math. 129 (1989) $1-60$.

[27] S.L. Richter and R.A. Decarlo, Continuation methods: Theory and Application. IEEE Trans. Circuits Syst. 30 (1983).

[28] E.D. Sontag, Mathematical Control Theory. Texts Appl. Math. 6, Springer-Verlag, New York, 2nd edition (1998).

[29] P. Souères and J.P. Laumond, Shortest paths synthesis for a car-like robot. IEEE Trans. Aut. Cont. 41 (1996) $672-688$.

[30] R. Strichartz, Sub-Riemannian Geometry. J. Diff. Geom. 24 (1983) 221-263.

[31] H.J. Sussmann, A Continuation Method for Nonholonomic Path-finding Problems, in Proceedings of the 32nd IEEE CDC, San Antonio, TX (Dec. 1993).

[32] H.J. Sussmann, New Differential Geometric Methods in Nonholonomic Path Finding, in Systems, Models, and Feedback, A. Isidori and T.J. Tarn Eds. Birkhäuser, Boston (1992).

[33] T. Wazewski, Sur l'évaluation du domaine d'existence des fonctions implicites réelles ou complexes. Ann. Soc. Polon. Math. 20 (1947). 